\title{
RELACIÓN ENTRE LA PERIMETRÍA AUTOMATIZADA CONVENCIONAL Y LA TOPOGRAFÍA PAPILAR REALIZADA CON EL TOMÓGRAFO HEIDELBERG
}

\author{
RELATIONSHIP BETWEEN STANDARD AUTOMATED \\ PERIMETRY AND OPTIC NERVE HEAD TOPOGRAPHY \\ PERFORMED WITH THE HEIDELBERG RETINA TOMOGRAPH
}

\author{
LÓPEZ-PEÑA MJ ${ }^{1}$, FERRERAS A ${ }^{2}$, LARROSA JM², POLO V ${ }^{2}$, FOGAGNOLO P ${ }^{3}$, HONRUBIA FM ${ }^{4}$
}

\section{RESUMEN}

Objetivo: Determinar las correlaciones entre los parámetros de la cabeza del nervio óptico $(\mathrm{CNO})$ obtenidos mediante el láser confocal de barrido (HRT), y los resultados de la perimetría automatizada convencional (PA) en sujetos normales, hipertensos oculares (HTO), sospechosos de glaucoma y glaucomatosos.

Métodos: Cuatrocientos veintitrés ojos fueron incluidos en el estudio y clasificados según la presión intraocular basal, morfología papilar y los resultados de la PA en 4 grupos: 87 normales, 192 hipertensos oculares, 70 sospechosos de glaucoma y 74 glaucomatosos. En los diferentes grupos diagnósticos, se calcularon los coeficientes de correlación de Pearson entre los parámetros de la $\mathrm{CNO}$ y la desviación media (DM), la desviación estándar de la media (DSM), el número de puntos alterados en cada cuadrante del campo visual (superior-nasal, inferior-nasal, superior-temporal e inferior-temporal), el número de puntos alterados según los niveles de probabilidad y los valores umbral en cada punto de la PA.

\begin{abstract}
Purpose: To determine the correlations between optic nerve head $(\mathrm{ONH})$ parameters measured with the Heidelberg Retina Tomograph (HRT), and the main outcomes of standard automated perimetry (SAP) in normal, ocular hypertensive, glaucoma suspects and glaucomatous subjects.

Methods: Four hundred and twenty-three patients were enrolled in the study and classified into four groups depending on baseline intraocular pressure, optic nerve head morphology, and SAP results: 87 normal eyes, 192 ocular hypertensive eyes, 70 glaucoma suspects and 74 glaucomatous eyes. In the different diagnostic groups, Pearson's correlation coefficients were calculated between $\mathrm{ONH}$ parameters and mean deviation, pattern standard deviation (PSD), number of altered points in each quadrant of the visual field (superior-nasal, inferior-nasal, superior-temporal and inferior-temporal), number of points altered at different probability levels, and threshold values at each point of SAP.

Results: In the normal and ocular hypertensive groups, only a few weak correlations were found
\end{abstract}

Recibido: 31/5/08. Aceptado: 17/12/09.

Servicio de Oftalmología. Hospital Universitario Miguel Servet. Zaragoza. España.

1 Doctor en Medicina. Servicio de Oftalmología. Hospital Universitario Miguel Servet. Zaragoza.

2 Doctor en Medicina. Servicio de Oftalmología. Hospital Universitario Miguel Servet. Instituto Aragonés de Ciencias de la Salud. Zaragoza.

Universidad de Zaragoza. Zaragoza.

3 Licenciado en Medicina. Servicio de Oftalmología. G.B. Bietti Foundation-IRCCS (Istituto de Ricovero e Cura a Carattere Scientifico). Roma. Italia.

4 Universidad de Zaragoza. Zaragoza.

Correspondencia:

María Jesús López Peña

Servicio de Oftalmología. Hospital Universitario Miguel Servet

$\mathrm{P}^{\circ}$ Isabel la Católica, 1-3

50009 Zaragoza

España

E-mail: mariaj.lopez@yahoo.com 
Resultados: En el grupo normal y de hipertensos oculares, se encontraron pocas correlaciones débiles entre los parámetros del HRT y los resultados de la PA. La fuerza y el número de correlaciones significativas aumentaron en el grupo de sospechosos de glaucoma. En el grupo de glaucomas, las correlaciones fueron más fuertes, especialmente entre los cocientes excavación/disco y anillo/disco con la DM $(r=0,479)$ y entre el área de anillo con la DSM $(\mathrm{r}=0,444)$.

Conclusiones: Se encontraron correlaciones débiles o moderadas entre algunos parámetros de la CNO medidos con el HRT y los resultados de la PA, en el grupo de glaucomas.

Palabras clave: Glaucoma, HRT, campo visual, cabeza nervio óptico. between HRT and SAP parameters. The strength and number of significant correlations increased in the suspected glaucoma group. The glaucoma group had the strongest correlations, particularly between cup/disc ratio and rim/disc ratio with MD $(\mathrm{r}=0.479)$ and between rim area and PSD $(\mathrm{r}=0.444)$. Conclusions: Weak to moderate correlations were found between some ONH parameters obtained with the HRT and SAP results in the glaucoma group (Arch Soc Esp Oftalmol 2009; 84: 611-624).

Key words: Glaucoma, HRT, visual field, optic nerve head.

\section{INTRODUCCIÓN}

El glaucoma primario de ángulo abierto se define como una neuropatía óptica progresiva que produce un daño en el nervio óptico y unos defectos característicos del campo visual (1-4). Suele ser bilateral aunque asimétrico, y en general, no da síntomas ni signos de alerta en la mayoría de los pacientes hasta estadios avanzados de la enfermedad, momento en el que existe un elevado riesgo de evolución a la ceguera irreversible $(3,5)$. Supone un problema importante de salud pública (5) que además representa la segunda causa de ceguera en el mundo (1).

Entre las diversas herramientas de estudio estructural en el glaucoma, se encuentra el Heidelberg retinal tomograph (HRT; Heidelberg Engineering, Heidelberg, Germany), que es un láser confocal de barrido que permite cuantificar diversos parámetros morfológicos de la papila. El explorador debe marcar los contornos del disco óptico, lo que en teoría podría incrementar la variabilidad externa, sin embargo, estudios comparativos con la planimetría muestran una mayor reproducibilidad del HRT (6).

Recientemente, Shunmugan y Azuara-Blanco (7) han recomendado el uso de las directrices del documento STARD (Standards for Reporting of Diagnostic Accuracy) para mejorar la calidad de las publicaciones sobre esta técnica. El propósito de la iniciativa STARD es mejorar la calidad de los estudios de habilidad diagnóstica. El diseño de este estudio se realizó de acuerdo a los 25 puntos definidos en la iniciativa STARD (8).
El objetivo de este estudio fue comparar los parámetros topográficos medidos con el HRT II con las principales variables de la perimetría automatizada convencional (PA) en diferentes grupos diagnósticos, para establecer la relación estructura-función entre ambas técnicas.

\section{SUJETOS, MATERIAL Y MÉTODO}

El diseño del estudio fue aceptado por el comité de ética del hospital, todos los participantes firmaron el consentimiento informado y la metodología del estudio se realizó siguiendo las directrices de la Declaración del Helsinki.

\section{Selección de la muestra}

Se pre-incluyeron un total de 435 sujetos desde junio de 2005 hasta junio de 2006. Dos de ellos no firmaron el consentimiento informado, en 3 no se obtuvieron resultados fiables en alguna de las pruebas realizadas y 7 no completaron las pruebas incluidas en el protocolo exploratorio. Finalmente, se incluyeron 423 sujetos de raza caucásica. Los criterios de inclusión fueron los siguientes: edad entre 30 y 75 años, agudeza visual mayor o igual a 20/30 (escala de Snellen), defectos de refracción menores de 5 dioptrías esféricas y astigmatismo menor de 3 dioptrías de equivalente esférico y medios ópticos transparentes (color u opalescencia nuclear, cortical 
Tabla I. Características clínicas de los grupos del estudio

\begin{tabular}{|c|c|c|c|c|c|c|c|c|c|c|c|c|}
\hline & \multicolumn{3}{|c|}{ Normales } & \multicolumn{3}{|c|}{ HTO } & \multicolumn{3}{|c|}{ Preperimétricos } & \multicolumn{3}{|c|}{ Glaucomas } \\
\hline & Media & DS & $\mathrm{p}$ & Media & DS & $\mathrm{p}$ & Media & DS & $\mathrm{p}$ & Media & DS & $\mathrm{p}$ \\
\hline Edad & 57,85 & 10,30 & & 58,66 & 10,42 & & 60,11 & 9,95 & & 61,96 & 7,29 & \\
\hline AV & 0,91 & 0,10 & $\mathrm{G}$ & 0,92 & 0,10 & $\mathrm{G}$ & 0,89 & 0,12 & $\mathrm{G}$ & 0,83 & 0,13 & $\mathrm{~N}, \mathrm{P}, \mathrm{H}$ \\
\hline PIO & 15,89 & 2,90 & $\mathrm{H}, \mathrm{P}, \mathrm{G}$ & 23,99 & 2,32 & $\mathrm{~N}$ & 23,50 & 2,40 & $\mathrm{~N}$ & 24,72 & 3,47 & $\mathrm{~N}$ \\
\hline E/D vertical & 0,29 & 0,15 & $\mathrm{H}, \mathrm{P}, \mathrm{G}$ & 0,43 & 0,16 & $\mathrm{~N}, \mathrm{P}, \mathrm{G}$ & 0,69 & 0,10 & $\mathrm{~N}, \mathrm{H}$ & 0,73 & 0,18 & $\mathrm{~N}, \mathrm{H}$ \\
\hline Paquimetría & 557,34 & 33,34 & $\mathrm{H}$ & 572,91 & 37,69 & $\mathrm{~N}, \mathrm{P}, \mathrm{G}$ & 550,47 & 32,91 & $\mathrm{H}$ & 543,74 & 36,04 & $\mathrm{H}$ \\
\hline Total & & 87 & & & 192 & & & 70 & & & 74 & \\
\hline
\end{tabular}

N: Diferencias con el grupo normal; H: Diferencias con el grupo de HTO; S: Diferencias con el grupo de sospechosos de glaucoma; G: Diferencias con el grupo de glaucomas; DS: desviación estándar; AV: mejor agudeza visual corregida; PIO: presión intraocular; E/D vertical: relación excavación/disco vertical en estereofotografías; HTO: hipertensos oculares. La columna p muestra los resultados de las ANOVAs.

o subcapsular posterior del cristalino $<1$ según el Lens Opacities Classification System III) (9). Se excluyeron aquellos sujetos con antecedentes de traumatismos, cirugía ocular previa, enfermedades sistémicas con repercusión oftálmica o incapacidad para la realización de alguna de las pruebas incluidas en el protocolo de estudio. Si ambos ojos eran elegibles, se seleccionó uno de forma aleatoria. A todos los participantes en el estudio se les realizó una exploración oftalmológica que incluyó: biomicroscopía con lámpara de hendidura, gonioscopía, medición de los niveles de presión intraocular (PIO)

Tabla II. Valores de los parámetros obtenidos mediante el HRT en cada uno de los grupos de estudio

\begin{tabular}{|c|c|c|c|c|c|c|c|c|c|c|c|c|}
\hline & \multicolumn{3}{|c|}{ Normales } & \multicolumn{3}{|c|}{ HTO } & \multicolumn{3}{|c|}{ Preperimétricos } & \multicolumn{3}{|c|}{ Glaucomas } \\
\hline & Media & DS & $\mathrm{p}$ & Media & DS & $\mathrm{p}$ & Media & DS & $\mathrm{p}$ & Media & DS & $\mathrm{p}$ \\
\hline Área disco & 1,96 & 0,33 & $\mathrm{P}, \mathrm{G}$ & 2,09 & 0,45 & $\mathrm{P}$ & 2,40 & 0,47 & $\mathrm{~N}, \mathrm{H}, \mathrm{G}$ & 2,18 & 0,42 & $\mathrm{P}, \mathrm{N}$ \\
\hline Área exc & 0,49 & 0,33 & $\mathrm{H}, \mathrm{P}, \mathrm{G}$ & 0,63 & 0,35 & $\mathrm{~N}, \mathrm{H}, \mathrm{P}$ & 1,13 & 0,38 & $\mathrm{~N}, \mathrm{H}$ & 1,15 & 0,47 & $\mathrm{~N}, \mathrm{H}$ \\
\hline Área anillo & 1,48 & 0,27 & $\mathrm{P}, \mathrm{G}$ & 1,45 & 0,28 & $\mathrm{P}, \mathrm{G}$ & 1,27 & 0,33 & $\mathrm{~N}, \mathrm{H}, \mathrm{G}$ & 1,02 & 0,31 & $\mathrm{~N}, \mathrm{H}, \mathrm{P}$ \\
\hline Área E/D & 0,24 & 0,13 & $\mathrm{H}, \mathrm{P}, \mathrm{G}$ & 0,29 & 0,13 & $\mathrm{~N}, \mathrm{H}, \mathrm{P}$ & 0,47 & 0,12 & $\mathrm{~N}, \mathrm{H}$ & 0,52 & 0,17 & $\mathrm{~N}, \mathrm{H}$ \\
\hline Área A/D & 0,76 & 0,13 & $\mathrm{H}, \mathrm{P}, \mathrm{G}$ & 0,71 & 0,13 & $\mathrm{~N}, \mathrm{H}, \mathrm{G}$ & 0,53 & 0,12 & $\mathrm{~N}, \mathrm{H}$ & 0,48 & 0,17 & $\mathrm{~N}, \mathrm{H}$ \\
\hline Vol exc & 0,12 & 0,13 & $\mathrm{P}, \mathrm{G}$ & 0,18 & 0,15 & $\mathrm{P}, \mathrm{G}$ & 0,35 & 0,18 & $\mathrm{~N}, \mathrm{H}$ & 0,37 & 0,28 & $\mathrm{~N}, \mathrm{H}$ \\
\hline Vol anillo & 0,41 & 0,13 & $\mathrm{P}, \mathrm{G}$ & 0,40 & 0,15 & $\mathrm{P}, \mathrm{G}$ & 0,31 & 0,14 & N,H,G & 0,22 & 0,12 & N,H,P \\
\hline Prof med exc & 0,22 & 0,09 & $\mathrm{H}, \mathrm{P}, \mathrm{G}$ & 0,27 & 0,10 & $\mathrm{~N}, \mathrm{P}, \mathrm{G}$ & 0,35 & 0,09 & $\mathrm{~N}, \mathrm{H}$ & 0,32 & 0,13 & $\mathrm{~N}, \mathrm{H}$ \\
\hline Prof máx exc & 0,62 & 0,24 & $\mathrm{P}$ & 0,70 & 0,23 & $\mathrm{P}$ & 0,80 & 0,20 & $\mathrm{~N}, \mathrm{H}$ & 0,72 & 0,25 & \\
\hline Var alt cont & 0,41 & 0,09 & & 0,42 & 0,11 & & 0,43 & 0,14 & & 0,44 & 0,26 & \\
\hline Morf exc & $-0,19$ & 0,07 & $\mathrm{H}, \mathrm{P}, \mathrm{G}$ & $-0,17$ & 0,07 & $\mathrm{~N}, \mathrm{P}, \mathrm{G}$ & $-0,09$ & 0,06 & $\mathrm{~N}, \mathrm{H}$ & $-0,08$ & 0,08 & $\mathrm{~N}, \mathrm{H}$ \\
\hline Esp med CFNR & 0,26 & 0,07 & $\mathrm{P}, \mathrm{G}$ & 0,26 & 0,07 & $\mathrm{P}, \mathrm{G}$ & 0,21 & 0,08 & $\mathrm{~N}, \mathrm{H}, \mathrm{G}$ & 0,15 & 0,09 & $\mathrm{~N}, \mathrm{H}, \mathrm{P}$ \\
\hline Sec CFNR & 1,29 & 0,32 & $\mathrm{G}$ & 1,31 & 0,38 & $\mathrm{G}$ & 1,17 & 0,49 & G & 0,75 & 0,50 & $\mathrm{~N}, \mathrm{H}, \mathrm{P}$ \\
\hline Coc hor E/D & 0,47 & 0,21 & $P, G$ & 0,52 & 0,18 & $\mathrm{P}, \mathrm{G}$ & 0,69 & 0,14 & $\mathrm{~N}, \mathrm{H}$ & 0,72 & 0,18 & $\mathrm{~N}, \mathrm{H}$ \\
\hline Coc vert E/D & 0,32 & 0,21 & $\mathrm{H}, \mathrm{P}, \mathrm{G}$ & 0,42 & 0,20 & $\mathrm{~N}, \mathrm{P}, \mathrm{G}$ & 0,64 & 0,12 & $\mathrm{~N}, \mathrm{H}$ & 0,66 & 0,20 & $\mathrm{~N}, \mathrm{H}$ \\
\hline Máx elev cont & $-0,12$ & 0,09 & $\mathrm{P}, \mathrm{G}$ & $-0,09$ & 0,09 & G & $-0,05$ & 0,09 & $\mathrm{~N}$ & $-0,02$ & 0,15 & $\mathrm{~N}, \mathrm{H}$ \\
\hline Máx dep cont & 0,29 & 0,13 & $\mathrm{P}, \mathrm{G}$ & 0,33 & 0,12 & $\mathrm{G}$ & 0,38 & 0,13 & $\mathrm{~N}$ & 0,42 & 0,25 & $\mathrm{~N}, \mathrm{H}$ \\
\hline LMC TS & 0,21 & 0,07 & $\mathrm{G}$ & 0,20 & 0,08 & $\mathrm{G}$ & 0,19 & 0,09 & $\mathrm{G}$ & 0,13 & 0,13 & $\mathrm{~N}, \mathrm{H}, \mathrm{P}$ \\
\hline LMC TI & 0,20 & 0,09 & $\mathrm{G}$ & 0,21 & 0,09 & $\mathrm{P}, \mathrm{G}$ & 0,15 & 0,13 & $\mathrm{H}, \mathrm{G}$ & 0,03 & 0,18 & N,H,P \\
\hline Variab media & 0,03 & 0,03 & $\mathrm{G}$ & 0,03 & 0,03 & $\mathrm{G}$ & 0,03 & 0,02 & $\mathrm{G}$ & 0,04 & 0,03 & $\mathrm{~N}, \mathrm{H}, \mathrm{P}$ \\
\hline Alt referencia & 0,32 & 0,13 & $\mathrm{P}$ & 0,36 & 0,11 & & 0,38 & 0,12 & $\mathrm{~N}$ & 0,32 & 0,13 & \\
\hline FSM & 1,55 & 1,52 & $P, G$ & 0,91 & 1,68 & $\mathrm{P}, \mathrm{G}$ & $-0,97$ & 1,72 & $\mathrm{~N}, \mathrm{H}, \mathrm{G}$ & $-2,04$ & 2,53 & $\mathrm{~N}, \mathrm{H}, \mathrm{P}$ \\
\hline RB & 1,53 & 0,73 & $\mathrm{P}, \mathrm{G}$ & 1,36 & 0,90 & $\mathrm{P}, \mathrm{G}$ & 0,63 & 0,99 & $\mathrm{~N}, \mathrm{H}, \mathrm{G}$ & $-0,27$ & 1,31 & $\mathrm{~N}, \mathrm{H}, \mathrm{P}$ \\
\hline
\end{tabular}

N: Diferencias con el grupo normal; H: Diferencias con el grupo de HTO; S: Diferencias con el grupo de sospechosos de glaucoma; G: Diferencias con el grupo de glaucomas; DS: Desviación estándar; HTO: Hipertensos oculares; Área exc: Área de excavación; Área E/D: Área excavación/disco; Área A/D: Área anillo/disco; Vol exc: Volumen de excavación, Vol anillo: Volumen de anillo; Prof med exc: Profundidad media de excavación; Prof máx exc: Profundidad máxima de excavación; Var alt cont: Variación altura de contorno; Morf exc: Morfología de la excavación; Esp med CFNR: Espesor medio de la capa de fibras nerviosas de la retina; Sec CFNR; Sección capa de fibras nerviosas de la retina; Coc hor E/D: Cociente horizontal excavación/disco; Coc vert E/D: Cociente vertical excavación/disco; Máx elev cont: Máxima elevación del contorno; Máx dep cont: Máxima depresión del contorno; LMC TS: Línea de modulación del contorno temporal superior; LMC TI: Línea de modulación del contorno temporal inferior; Variab media: Variabilidad media; Alt referencia: Altura de referencia; FSM: Función discriminante lineal de Mikelberg FS; RB: Función discriminante lineal de Burk RO. La columna p muestra los resultados de las ANOVAs. 
Tabla III. Correlaciones entre los índices de la perimetría, el número de puntos alterados por cuadrantes y el número de puntos alterados por niveles de probabilidad con los parámetros de la CNO evaluados con el HRT en el grupo de sujetos normales. Las correlaciones significativas $(\mathbf{p}<0,05)$ están resaltadas en fondo gris

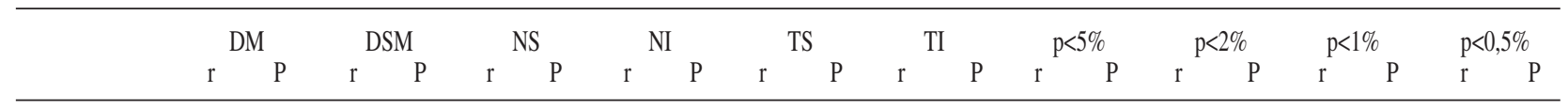

Área disco

Área excav.

Área anillo

$\mathrm{Coc} \mathrm{E} / \mathrm{D}$

$\mathrm{Coc} \mathrm{A} / \mathrm{D}$

Volumen exc

Volumen anillo

Prf med exc

Prf máx exc

Var alt cnt

Morf excav.

Esp med CFNR

Sección CFNR

Coc E/D hor

Coc E/D vert

Máx elev cont

Máx dep cont

LMC TS

LMC TI

Variab med

Altura ref.

FDL FSM

FDL RB

Dx Moorfields

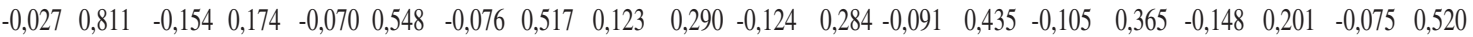
$\begin{array}{llllllllllllllllllll}0,154 & 0,168 & -0,298 & 0,007 & -0,301 & 0,008 & -0,165 & 0,155 & 0,030 & 0,800 & 0,013 & 0,915 & -0,215 & 0,062 & -0,107 & 0,357 & -0,068 & 0,561 & 0,030 & 0,800\end{array}$

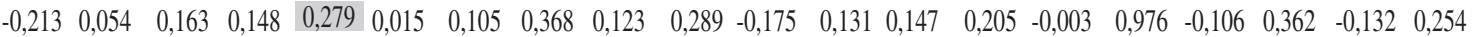
$\begin{array}{llllllllllllllllllll}0,174 & 0,118 & -0,323 & 0,003 & -0,336 & 0,003 & -0,181 & 0,118 & 0,021 & 0,859 & 0,024 & 0,838 & -0,237 & 0,039 & -0,107 & 0,357 & -0,034 & 0,774 & 0,051 & 0,661\end{array}$ $\begin{array}{llllllllllllllllllll}-0,174 & 0,118 & 0,323 & 0,003 & 0,336 & 0,003 & 0,181 & 0,118 & -0,021 & 0,859 & -0,024 & 0,838 & 0,237 & 0,039 & 0,107 & 0,357 & 0,034 & 0,774 & -0,051 & 0,661\end{array}$ $\begin{array}{llllllllllllllllllll}0,145 & 0,194 & -0,192 & 0,088 & -0,221 & 0,055 & -0,081 & 0,484 & 0,006 & 0,961 & 0,083 & 0,478 & -0,111 & 0,342 & -0,023 & 0,843 & 0,013 & 0,908 & 0,051 & 0,663\end{array}$ $\begin{array}{llllllllllllllllllll}-0,297 & 0,007 & 0,152 & 0,179 & 0,283 & 0,013 & 0,144 & 0,215 & 0,066 & 0,574 & -0,158 & 0,172 & 0,164 & 0,156 & 0,089 & 0,444 & 0,018 & 0,878 & -0,056 & 0,628\end{array}$ $\begin{array}{llllllllllllllllllll}0,052 & 0,645 & -0,195 & 0,083 & -0,209 & 0,070 & -0,042 & 0,719 & 0,069 & 0,553 & 0,054 & 0,645 & -0,077 & 0,511 & 0,064 & 0,584 & 0,114 & 0,328 & 0,079 & 0,496\end{array}$ $\begin{array}{llllllllllllllllllll}0,017 & 0,878 & -0,017 & 0,883 & -0,087 & 0,457 & 0,096 & 0,408 & 0,078 & 0,503 & 0,107 & 0,358 & 0,080 & 0,490 & 0,179 & 0,122 & 0,233 & 0,043 & 0,056 & 0,633\end{array}$ $\begin{array}{llllllllllllllllllll}-0,052 & 0,641 & -0,038 & 0,737 & 0,080 & 0,490 & 0,055 & 0,639 & -0,021 & 0,854 & -0,202 & 0,080 & -0,013 & 0,912 & -0,109 & 0,348 & -0,115 & 0,323 & -0,131 & 0,260\end{array}$ $\begin{array}{llllllllllllllllllll}-0,020 & 0,859 & -0,413 & 0,000 & -0,184 & 0,111 & -0,250 & 0,029 & 0,034 & 0,771 & -0,139 & 0,232 & -0,272 & 0,017 & -0,161 & 0,165 & -0,160 & 0,167 & 0,058 & 0,618\end{array}$ $\begin{array}{llllllllllllllllllll}-0,144 & 0,197 & 0,001 & 0,994 & 0,090 & 0,437 & 0,127 & 0,275 & -0,069 & 0,552 & -0,055 & 0,638 & 0,078 & 0,505 & 0,023 & 0,843 & -0,029 & 0,803 & -0,082 & 0,481\end{array}$

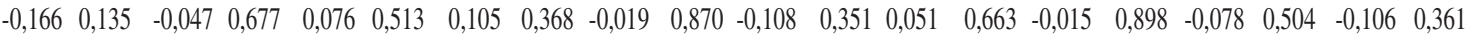
$\begin{array}{llllllllllllllllllll}0,185 & 0,097 & -0,293 & 0,008 & -0,205 & 0,076 & -0,094 & 0,418 & 0,066 & 0,570 & -0,001 & 0,994 & -0,128 & 0,272 & -0,060 & 0,606 & 0,002 & 0,989 & 0,025 & 0,832\end{array}$ $\begin{array}{llllllllllllllllllll}0,127 & 0,254 & -0,250 & 0,025 & -0,351 & 0,002 & -0,104 & 0,372 & 0,105 & 0,368 & 0,014 & 0,902 & -0,180 & 0,120 & 0,010 & 0,932 & 0,076 & 0,513 & 0,106 & 0,364\end{array}$ $\begin{array}{llllllllllllllllllll}0,104 & 0,353 & -0,217 & 0,054 & -0,137 & 0,239 & -0,131 & 0,258 & -0,082 & 0,480 & -0,029 & 0,802 & -0,168 & 0,146 & -0,012 & 0,916 & 0,054 & 0,641 & -0,010 & 0,930\end{array}$ $\begin{array}{lllllllllllllllllllllllll}0,038 & 0,735 & -0,186 & 0,098 & -0,040 & 0,729 & -0,055 & 0,636 & -0,076 & 0,512 & -0,168 & 0,148 & -0,131 & 0,258 & -0,089 & 0,447 & -0,047 & 0,689 & -0,103 & 0,375\end{array}$ $\begin{array}{lllllllllllllllllllllllll}-0,152 & 0,174 & 0,002 & 0,987 & 0,049 & 0,675 & 0,011 & 0,927 & -0,040 & 0,734 & -0,098 & 0,400 & -0,019 & 0,872 & -0,083 & 0,478 & -0,148 & 0,202 & -0,243 & 0,034\end{array}$ $\begin{array}{llllllllllllllllllllll}-0,002 & 0,984 & 0,023 & 0,841 & 0,113 & 0,329 & 0,186 & 0,108 & -0,089 & 0,445 & -0,119 & 0,308 & 0,095 & 0,416 & 0,050 & 0,668 & 0,016 & 0,889 & 0,053 & 0,648\end{array}$ $\begin{array}{lllllllllllllllllllllllll}-0,176 & 0,113 & -0,324 & 0,003 & 0,015 & 0,898 & -0,190 & 0,101 & 0,131 & 0,258 & 0,041 & 0,726 & -0,061 & 0,601 & -0,051 & 0,663 & -0,039 & 0,741 & -0,023 & 0,844\end{array}$ $\begin{array}{llllllllllllllllllll}0,030 & 0,791 & -0,167 & 0,138 & -0,033 & 0,778 & -0,021 & 0,855 & -0,079 & 0,495 & -0,124 & 0,287 & -0,092 & 0,429 & -0,042 & 0,716 & -0,023 & 0,841 & -0,085 & 0,463\end{array}$ $\begin{array}{lllllllllllllllllllll}-0,234 & 0,035 & 0,420 & 0,000 & 0,360 & 0,001 & 0,264 & 0,021 & 0,080 & 0,493 & 0,002 & 0,987 & 0,332 & 0,003 & 0,246 & 0,033 & 0,190 & 0,100 & 0,001 & 0,992\end{array}$ $\begin{array}{llllllllllllllllllll}-0,054 & 0,627 & 0,226 & 0,044 & 0,242 & 0,035 & 0,229 & 0,046 & -0,077 & 0,507 & -0,035 & 0,761 & 0,209 & 0,070 & 0,112 & 0,335 & 0,012 & 0,918 & -0,045 & 0,698\end{array}$ $\begin{array}{lllllllllllllllllllll}-0,016 & 0,884 & -0,304 & 0,006 & -0,107 & 0,358 & -0,112 & 0,336 & 0,053 & 0,648 & -0,003 & 0,983 & -0,101 & 0,387 & -0,038 & 0,746 & 0,028 & 0,812 & -0,048 & 0,682\end{array}$

r: Coeficiente de regresión de Pearson; P: Nivel de significación estadística ( $<<0,05)$; NS: Número de puntos alterados en el cuadrante nasal-superior; NI: Número de puntos alterados en el cuadrante nasal-inferior; TS: Número de puntos alterados en el cuadrante temporal-superior; TI: Número de puntos alterados en el cuadrante temporal-inferior; $\mathrm{P}<5 \%$ : Número de puntos con un nivel de significación $\mathrm{p}<5 \%$; $\mathrm{P}<2 \%$ : Número de puntos con un nivel de significación $\mathrm{p}<2 \% ; \mathrm{P}<1 \%$ : Número de puntos con un nivel de significación $\mathrm{p}<1 \%$; $\mathrm{P}<0,5 \%:$ : Número de puntos con un nivel de significación p<0,5\%; Área exc: Área de excavación; Área E/D: Área excavación/disco; Área A/D: Área anillo/disco; Vol exc: Volumen de excavación, Vol anillo: Volumen de anillo; Prof med exc: Profundidad media de excavación; Prof máx exc: Profundidad máxima de excavación; Var alt cont: Variación altura de contorno; Morf exc: Morfología de la excavación; Esp med CFNR: Espesor medio de la capa de fibras nerviosas de la retina; Sec CFNR; Sección capa de fibras nerviosas de la retina; Coc hor E/D: Cociente horizontal excavación/disco; Coc vert E/D: Cociente vertical excavación/disco; Máx elev cont: Máxima elevación del contorno; Máx dep cont: Máxima depresión del contorno; LMC TS: Línea de modulación del contorno temporal superior; LMC TI: Línea de modulación del contorno temporal inferior; Variab media: Variabilidad media; Alt referencia: Altura de referencia; FSM: Función discriminante lineal de Mikelberg FS; RB: Función discriminante lineal de Burk RO; Dx Moorfields: Diagnóstico del Análisis de regresión de Moorfields.

mediante tonometría de aplanación, paquimetría corneal central (modelo DGH 500; DGH Technology, Exton, PE, EEUU) fondo de ojo mediante oftalmoscopía indirecta, estereofotografías papilares, al menos 2 PAs, y una topografía papilar con el HRT II.

\section{Metodología de las pruebas complementarias}

La evaluación clínica del nervio óptico fue realizada a partir de las estereofotografías papilares por dos especialistas en glaucoma (AF y JML) enmascarados con respecto a la historia clínica del sujeto. Las discrepancias entre observadores fueron resueltas por consenso. Se definió la apariencia del nervio óptico compatible con glaucoma como la existencia de un adelgazamiento del anillo neurorretiniano focal (presencia de muescas) o difuso con aumento de la excavación.

Las PAs se realizaron con el Analizador de campo Humphrey modelo 750 (Zeiss Humphrey Systems, Dublín, CA, EEUU) y la estrategia 24-2 
Tabla IV. Correlaciones entre los índices de la perimetría, el número de puntos alterados por cuadrantes y el número de puntos alterados por niveles de probabilidad con los parámetros de la CNO evaluados con el HRT en el grupo de hipertensos oculares. Las correlaciones significativas $(\mathbf{p}<0,05)$ están resaltadas en fondo gris

\begin{tabular}{|c|c|c|c|c|c|c|c|c|c|c|c|c|c|c|c|c|c|c|c|c|}
\hline & \multicolumn{2}{|c|}{ DM } & \multicolumn{2}{|c|}{ DSM } & \multicolumn{2}{|c|}{ NS } & \multicolumn{2}{|c|}{ NI } & \multicolumn{2}{|c|}{ TS } & \multicolumn{2}{|c|}{ TI } & \multicolumn{2}{|c|}{$\mathrm{p}<5 \%$} & \multicolumn{2}{|c|}{$\mathrm{p}<2 \%$} & \multicolumn{2}{|c|}{$\mathrm{p}<1 \%$} & \multicolumn{2}{|c|}{$\mathrm{p}<0,5 \%$} \\
\hline & $\mathrm{r}$ & P & $\mathrm{r}$ & $\mathrm{P}$ & $\mathrm{r}$ & $\mathrm{P}$ & $\mathrm{r}$ & P & $\mathrm{r}$ & $\mathrm{P}$ & $\mathrm{r}$ & $P$ & $\mathrm{r}$ & $\mathrm{P}$ & $\mathrm{r}$ & $\mathrm{P}$ & $\mathrm{r}$ & $\mathrm{P}$ & $\mathrm{r}$ & $\mathrm{P}$ \\
\hline Área disco & 0,078 & 0,285 & $-0,038$ & 0,601 & $-0,192$ & 0,009 & $-0,030$ & 0,691 & 0,016 & 0,834 & 0,011 & 0,880 & $-0,091$ & 0,222 & $-0,088$ & 0,238 & $-0,006$ & 0,941 & 0,030 & 0,682 \\
\hline Área excav. & 0,116 & 0,108 & $-0,056$ & 0,444 & $-0,171$ & 0,020 & 0,005 & 0,945 & 0,026 & 0,725 & 0,040 & 0,593 & $-0,055$ & 0,462 & $-0,041$ & 0,582 & $-0,025$ & 0,737 & 0,020 & 0,791 \\
\hline Área anillo & $-0,022$ & 0,757 & 0,012 & 0,867 & $-0,090$ & 0,227 & $-0,049$ & 0,513 & $-0,009$ & 0,904 & $-0,032$ & 0,664 & $-0,073$ & 0,324 & $-0,085$ & 0,249 & 0,024 & 0,748 & 0,023 & 0,760 \\
\hline Coc E/D & 0,098 & 0,175 & $-0,026$ & 0,715 & $-0,128$ & 0,083 & 0,031 & 0,672 & 0,065 & 0,381 & 0,045 & 0,545 & $-0,009$ & 0,908 & 0,000 & 0,999 & $-0,019$ & 0,798 & 0,018 & 0,812 \\
\hline $\mathrm{Coc} \mathrm{A} / \mathrm{D}$ & $-0,098$ & 0,175 & 0,026 & 0,715 & 0,128 & 0,083 & $-0,031$ & 0,672 & $-0,065$ & 0,381 & $-0,045$ & 0,545 & 0,009 & 0,908 & 0,000 & 0,999 & 0,019 & 0,798 & $-0,018$ & 0,812 \\
\hline Volumen exc & 0,124 & 0,087 & $-0,074$ & 0,311 & $-0,174$ & 0,018 & $-0,046$ & 0,532 & 0,027 & 0,719 & 0,096 & 0,197 & $-0,059$ & 0,425 & $-0,032$ & 0,665 & $-0,031$ & 0,672 & 0,001 & 0,994 \\
\hline Volumen anillo & $-0,075$ & 0,304 & $-0,001$ & 0,985 & $-0,063$ & 0,394 & $-0,059$ & 0,429 & $-0,074$ & 0,315 & $-0,095$ & 0,19 & $-0,108$ & 0,143 & $-0,114$ & 0,124 & $-0,065$ & 0,383 & $-0,055$ & 0,461 \\
\hline Prf med exc & 0,079 & 0,275 & $-0,049$ & 0,496 & $-0,136$ & 0,066 & $-0,014$ & 0,848 & 0,061 & 0,407 & 0,070 & 0,348 & $-0,024$ & 0,747 & 0,001 & 0,987 & $-0,042$ & 0,576 & $-0,022$ & 0,766 \\
\hline Prf máx exc & 0,012 & 0,873 & $-0,073$ & 0,317 & $-0,115$ & 0,121 & $-0,081$ & 0,272 & 0,069 & 0,351 & 0,029 & 0,700 & $-0,050$ & 0,502 & $-0,055$ & 0,462 & $-0,078$ & 0,295 & $-0,046$ & 0,534 \\
\hline Var alt cnt & $-0,108$ & 0,137 & 0,003 & 0,967 & $-0,041$ & 0,585 & $-0,022$ & 0,768 & $-0,123$ & 0,098 & $-0,095$ & 0,201 & $-0,101$ & 0,171 & $-0,095$ & 0,198 & $-0,098$ & 0,187 & $-0,105$ & 0,156 \\
\hline Morf excav. & 0,130 & 0,072 & 0,050 & 0,492 & 0,011 & 0,877 & 0,160 & 0,031 & 0,032 & 0,669 & 0,091 & 0,220 & 0,107 & 0,149 & 0,141 & 0,056 & 0,077 & 0,300 & 0,060 & 0,422 \\
\hline Esp med CFNR & $-0,072$ & 0,318 & $-0,024$ & 0,739 & 0,007 & 0,921 & $-0,060$ & 0,415 & $-0,089$ & 0,229 & $-0,093$ & 0,211 & $-0,082$ & 0,271 & $-0,083$ & 0,263 & $-0,104$ & 0,162 & $-0,089$ & 0,232 \\
\hline Sección CFNR & $-0,039$ & 0,593 & $-0,034$ & 0,636 & $-0,066$ & 0,374 & $-0,058$ & 0,436 & $-0,080$ & 0,281 & $-0,096$ & 0,196 & $-0,111$ & 0,132 & $-0,117$ & 0,114 & $-0,104$ & 0,159 & $-0,067$ & 0,363 \\
\hline Coc E/D hor & $-0,025$ & 0,730 & $-0,073$ & 0,316 & $-0,113$ & 0,127 & $-0,016$ & 0,828 & 0,041 & 0,583 & 0,036 & 0,62 & $-0,032$ & 0,667 & $-0,038$ & 0,605 & $-0,048$ & 0,513 & $-0,023$ & 0,758 \\
\hline Coc E/D vert & 0,068 & 0,349 & $-0,013$ & 0,859 & $-0,080$ & 0,283 & 0,078 & 0,290 & 0,134 & 0,069 & 0,038 & & & 0,453 & 0,040 & 0,591 & 0,017 & 0,822 & 0,011 & 0,879 \\
\hline Máx elev cont & 0,121 & 0,093 & $-0,067$ & 0,356 & 0,057 & 0,442 & $-0,063$ & 0,398 & 0,089 & 0,231 & 0,085 & 0,250 & 0,060 & 0,415 & 0,009 & 0,901 & $-0,011$ & 0,886 & $-0,007$ & 0,925 \\
\hline Máx dep cont & $-0,015$ & 0,836 & $-0,044$ & 0,541 & 0,001 & 0,986 & $-0,064$ & 0,390 & $-0,053$ & 0,471 & $-0,030$ & 0,691 & $-0,053$ & 0,473 & $-0,083$ & 0,265 & $-0,099$ & 0,182 & $-0,103$ & 0,164 \\
\hline LMC TS & $-0,064$ & 0,377 & $-0,012$ & 0,871 & 0,026 & 0,728 & $-0,009$ & 0,907 & $-0,021$ & 0,772 & $-0,015$ & 0,837 & $-0,004$ & 0,955 & 0,008 & 0,913 & 0,012 & 0,873 & 0,006 & 0,932 \\
\hline LMC TI & $-0,112$ & 0,123 & $-0,069$ & 0,341 & $-0,033$ & 0,653 & $-0,096$ & 0,193 & $-0,098$ & 0,186 & $-0,031$ & 0,677 & $-0,099$ & 0,182 & $-0,139$ & 0,060 & $-0,191$ & 0,009 & $-0,131$ & 0,075 \\
\hline Variab med & $-0,196$ & 0,006 & 0,071 & 0,326 & 0,027 & 0,718 & 0,053 & 0,471 & 0,038 & 0,608 & 0,060 & 0,417 & 0,066 & 0,377 & 0,038 & 0,604 & 0,057 & 0,443 & 0,029 & 0,698 \\
\hline Altura ref. & $-0,001$ & 0,990 & $-0,057$ & 0,429 & 0,017 & 0,820 & $-0,074$ & 0,317 & $-0,055$ & 0,457 & $-0,022$ & 0,770 & $-0,048$ & 0,515 & $-0,080$ & 0,279 & $-0,111$ & 0,134 & $-0,103$ & 0,165 \\
\hline FDL FSM & $-0,099$ & 0,172 & 0,017 & 0,819 & $-0,012$ & 0,874 & $-0,094$ & 0,205 & 0,028 & 0,704 & $-0,059$ & 0,427 & $-0,049$ & 0,506 & $-0,093$ & 0,211 & $-0,025$ & 0,737 & $-0,030$ & 0,689 \\
\hline FDL RB & $-0,183$ & 0,011 & $-0,064$ & 0,380 & $-0,001$ & 0,986 & $-0,126$ & 0,089 & $-0,075$ & 0,309 & $-0,059$ & 0,427 & $-0,096$ & 0,196 & $-0,106$ & 0,151 & $-0,105$ & 0,154 & $-0,088$ & 0,236 \\
\hline Dx Moorfields & $-0,168$ & 0,020 & 0,045 & 0,533 & $-0,101$ & 0,172 & 0,075 & 0,312 & 0,011 & 0,886 & 0,048 & 0,515 & 0,001 & 0,984 & 0,025 & 0,734 & 0,003 & 0,963 & $-0,052$ & 0,479 \\
\hline
\end{tabular}

r: Coeficiente de regresión de Pearson; P: Nivel de significación estadística ( $<<0,05)$; NS: Número de puntos alterados en el cuadrante nasal-superior; NI: Número de puntos alterados en el cuadrante nasal-inferior; TS: Número de puntos alterados en el cuadrante temporal-superior; TI: Número de puntos alterados en el cuadrante temporal-inferior; $\mathrm{P}<5 \%$ : Número de puntos con un nivel de significación $\mathrm{p}<5 \%$; $\mathrm{P}<2 \%$ : Número de puntos con un nivel de significación $\mathrm{p}<2 \% ; \mathrm{P}<1 \%$ : Número de puntos con un nivel de significación $\mathrm{p}<1 \% ; \mathrm{P}<0,5 \%$ : Número de puntos con un nivel de significación p<0,5\%; Área exc: Área de excavación; Área E/D: Área excavación/disco; Área A/D: Área anillo/disco; Vol exc: Volumen de excavación, Vol anillo: Volumen de anillo; Prof med exc: Profundidad media de excavación; Prof máx exc: Profundidad máxima de excavación; Var alt cont: Variación altura de contorno; Morf exc: Morfología de la excavación; Esp med CFNR: Espesor medio de la capa de fibras nerviosas de la retina; Sec CFNR; Sección capa de fibras nerviosas de la retina; Coc hor E/D: Cociente horizontal excavación/disco; Coc vert E/D: Cociente vertical excavación/disco; Máx elev cont: Máxima elevación del contorno; Máx dep cont: Máxima depresión del contorno; LMC TS: Línea de modulación del contorno temporal superior; LMC TI: Línea de modulación del contorno temporal inferior; Variab media: Variabilidad media; Alt referencia: Altura de referencia; FSM: Función discriminante lineal de Mikelberg FS; RB: Función discriminante lineal de Burk RO; Dx Moorfields: Diagnóstico del Análisis de regresión de Moorfields.

SITA Standard. Se siguieron los criterios de fiabilidad establecidos por el propio aparato. La PA se consideró anormal cuando en al menos 2 pruebas aparecieron defectos reproducibles no explicables por otra causa que no fuera el glaucoma y definidos por la presencia de 3 puntos contiguos con un nivel de significación $\mathrm{p}<0,05$ en el mapa de desviación de patrón, o 2 con una $p<0,01$, o una desviación estándar de la media (DSM) con una $\mathrm{p}<0,05$, o una prueba del hemicampo glaucomatoso fuera de límites normales. Se realizaron al menos 2 PAs para disminuir el efecto aprendizaje. Los parámetros perimétricos correlacionados fueron: la desviación media (DM), la desviación estándar de la media (DSM), el número de puntos alterados en cada cuadrante del campo visual: nasal-superior (NS), nasal-inferior (NI), temporal-superior (TS) y temporal-inferior (TI), el número de puntos alterados en cada nivel de probabilidad $(5 \%, 2 \%, 1 \%$ y $0,5 \%)$ y los valores umbral de cada uno de los 52 puntos de la PA. 
Tabla V. Correlaciones entre los índices de la perimetría, el número de puntos alterados por cuadrantes y el número de puntos alterados por niveles de probabilidad con los parámetros de la CNO evaluados con el HRT en el grupo de sujetos con glaucoma pre-perimétrico. Las correlaciones significativas $(\mathbf{p}<0,05)$ están resaltadas en fondo gris

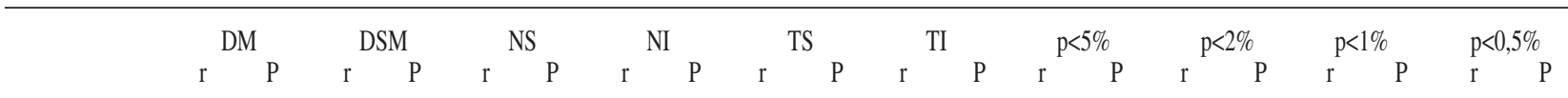

\begin{tabular}{|c|c|c|c|c|c|c|c|c|c|c|c|c|c|c|c|c|c|c|}
\hline Área disco & 0,032 & 0,795 & $-0,120$ & 0,321 & 0,122 & 0,356 & $-0,289$ & 0,027 & 0,031 & $0,8160,262$ & $0,0450,058$ & 0,662 & $-0,027$ & 0,842 & 0,042 & 0,749 & $-0,009$ & 0,944 \\
\hline Área excav. &, 157 & 0,195 & 0,009 & 0,943 & 0,152 & 251 & $-0,140$ & 0,291 & 0,092 & $0,488 \quad 0,262$ & $\begin{array}{lll}0,045 & 0,182\end{array}$ & 0,169 & 0,050 & 0,710 & 0,076 & 0,568 & 0,027 & 0,841 \\
\hline Área anillo & 0,224 & 0,062 & $-0,180$ & 137 & 0,016 & 0,905 & $-0,274$ & 036 & $-0,054$ & $0,686 \quad 0,103$ & $437-0,110$ & 0,407 & $-0,092$ & 0,487 & $-0,019$ & 0,885 & $-0,042$ & \\
\hline Coc E/D & $-0,240$ & 0,045 & 0,099 & 0,413 & 0,113 & 0,393 & 0,090 & 0,499 & 0,120 & $\begin{array}{lll}0,364 & 0,124\end{array}$ & 5510,226 & 0,085 & 0,103 & 0,438 & 0,054 & 0,685 & 0,038 & 0,779 \\
\hline $\mathrm{Coc} \mathrm{A} / \mathrm{D}$ & 0,240 & 045 & $-0,099$ &, 413 & $-0,11$ & 393 & $-0,090$ & 0,499 & $-0,120$ & $\begin{array}{lll}0,364 & -0,124\end{array}$ & $1-0,226$ & 0,085 & $-0,103$ & 438 & $-0,054$ & 685 & $-0,038$ & 0,779 \\
\hline Volumen exc & $-0,182$ & 0,132 & 0,108 & 0,371 & 0,096 & 0,468 & 0,063 & 0,636 & 0,065 & $0,627 \quad 0,295$ & 30,265 & 0,043 & 0,074 & ,579 & $-0,041$ & 0,759 & $-0,150$ &, 261 \\
\hline Volumen anillo & 0,242 & 0,044 & $-0,129$ & 0,288 & $-0,080$ & 0,545 & $-0,171$ & 0,195 & 0,019 & $\begin{array}{lll}0,885 & -0,047\end{array}$ & $0,723-0,147$ & 0,267 & $-0,158$ & 0,233 & $-0,089$ & 0,504 & $-0,081$ & 0,546 \\
\hline Prf med exc & $-0,078$ & 0,523 & 0,191 & 0,113 & 0,084 & 0,528 & 0,117 & 0,377 & 0,066 & 0,153 & $0,248 \quad 0,215$ & 0,102 & 0,006 & 966 & $-0,100$ & 451 & $-0,213$ & 108 \\
\hline Prf máx exc & $-0,152$ & 0,208 & 0,218 & 0,069 & 0,071 & 0,591 & 0,140 & 0,291 & 0,012 & $0,931 \quad 0,166$ & $0,208 \quad 0,201$ & 0,126 & 0,053 & 0,691 & $-0,049$ & 0,715 & $-0,141$ & 0,290 \\
\hline Var alt cnt & $-0,057$ & 0,637 & 0,233 & 0,052 & $-0,103$ & 0,439 & $-0,028$ & 0,830 & 0,157 & $0,235 \quad 0,102$ & $0,443 \quad 0,060$ & 0,651 & 0,060 & 0,650 & $-0,020$ & 0,882 & 0,127 & 0,344 \\
\hline Morf excav. & $-0,092$ & 0,450 & 0,056 & 0,646 & 0,028 & 0,833 & 0,014 & 0,915 & 0,228 & $0,082 \quad 0,123$ & $0,353 \quad 0,194$ & 0,140 & 0,095 & 0,476 & 0,054 & 0,683 & $-0,036$ & 0,101 \\
\hline Esp med CFNR & 0,292 & 0,014 & $-0,041$ & 0,735 & 0,026 & 0,848 & $-0,155$ & 0,242 & 0,018 & $-0,227$ & $0,084-0,177$ & 0,180 & $-0,197$ & 35 & $-0,102$ & 0,444 & $-0,047$ & 0,728 \\
\hline Sección CFNR & 0,27 & 0,023 & $-0,067$ & 0,584 & 0,059 & 0,657 & $-0,207$ & 0,116 & 0,033 & $-0,141$ & $0,288-0,136$ & 0,304 & $-0,176$ & & $-0,084$ & 0,525 & $-0,043$ &, 746 \\
\hline Coc E/D hor & $-0,184$ & 0,128 & 0,103 & 0,394 & 0,095 & 0,474 & $-0,026$ & 0,843 & 0,055 & $\begin{array}{lll}0,677 & 0,085\end{array}$ & $\begin{array}{lll}0,520 & 0,105\end{array}$ & 0,430 & 0,016 & 0,904 & $-0,133$ & 0,314 & $-0,092$ & 0,490 \\
\hline Coc E/D vert & $-0,235$ & 0,050 & 0,146 & 0,229 & 0,053 & 0,691 & 0,313 & 0,016 & 0,109 & $0,411 \quad 0,052$ & $0,693 \quad 0,272$ & 0,037 & 0,242 & 0,065 & 0,284 & 0,029 & 0,094 & 0,482 \\
\hline Máx elev cont & 0,106 & 0,384 & $-0,046$ & 0,708 & 0,094 & 0,480 & 0,083 & 0,532 & $-0,052$ & $0,697-0,016$ & $\begin{array}{lll}0,907 & 0,059\end{array}$ & 0,657 & 0,060 & 0,650 & 0,025 & 0,849 & 0,156 & 0,242 \\
\hline Máx dep cont & 0,012 & 0,920 & 0,217 & 0,071 & $-0,045$ & 0,736 & 0,026 & 0,844 & 0,131 & $0,323 \quad 0,097$ & $0,463 \quad 0,104$ & 0,434 & 0,104 & 0,433 & $-0,004$ & 0,975 & 0,241 & 0,069 \\
\hline LMC TS & $-0,098$ & 0,422 & 0,236 & 0,049 & 0,162 & 0,221 & $-0,115$ & 0,386 & 0,002 & $\begin{array}{ll}0,986 & 0,026\end{array}$ & $\begin{array}{lll}0,847 & 0,036\end{array}$ & 0,789 & $-0,093$ & 0,483 & 0,050 & 0,709 & 0,052 & 0,699 \\
\hline LMC TI & 0,40 & 0,001 & $-0,209$ & 0,082 & $-0,15$ & 0,250 & $-0,130$ & 0,326 & $-0,072$ & $0,587-0,160$ & $0,226-0,263$ & 0,044 & $-0,193$ & 0,142 & $-0,217$ & 0,098 & $-0,152$ & 0,255 \\
\hline Variab med & $-0,298$ & 0,012 & 0,064 & 0,597 & $-0,01$ & 0,932 & 0,119 & 0,368 & 0,006 & $0,965 \quad 0,007$ & $\begin{array}{lll}0,956 & 0,064\end{array}$ & 0,629 & 0,073 & 0,584 & 0,008 & 0,949 & $-0,043$ & 0,749 \\
\hline Altura ref. & 0,210 & 0,082 & 0,070 & 0,566 & 0,031 & 0,815 & $-0,061$ & 0,645 & 0,088 & $0,505-0,070$ & 0,599-0,010 & 0,939 & $-0,024$ & 0,857 & $-0,026$ & 0,847 & 0,148 & 0,266 \\
\hline FDL FSM & 0,251 & 0,036 & $-0,252$ & 0,036 & 0,003 & 0,979 & $-0,101$ & 0,445 & $-0,194$ & $0,140-0,160$ & $0,226-0,227$ & 0,084 & $-0,195$ & 0,140 & $-0,049$ & 0,713 & $-0,114$ & 0,395 \\
\hline FDL RB & 0,269 & 0,024 & $-0,074$ & 0,540 & 0,002 & 0,986 & $-0,115$ & 0,386 & $-0,106$ & $0,423-0,120$ & $0,366-0,172$ & 0,192 & $-0,184$ & 0,164 & $-0,119$ & 0,370 & $-0,059$ & 0,658 \\
\hline Dx Moorfields & $-0,058$ & 0,632 & 0,019 & 0,874 & 0,001 & 0,995 & 0,068 & 0,609 & 0,097 & $0,464-0,056$ & $0,675 \quad 0,054$ & 0,682 & 0,062 & 0,641 & 0,170 & 0,199 & 0,191 & 0,151 \\
\hline
\end{tabular}

r: Coeficiente de regresión de Pearson; P: Nivel de significación estadística ( $\mathrm{p}<0,05)$; NS: Número de puntos alterados en el cuadrante nasal-superior; NI: Número de puntos alterados en el cuadrante nasal-inferior; TS: Número de puntos alterados en el cuadrante temporal-superior; TI: Número de puntos alterados en el cuadrante temporal-inferior; $\mathrm{P}<5 \%$ : Número de puntos con un nivel de significación $\mathrm{p}<5 \%$; $\mathrm{P}<2 \%$ : Número de puntos con un nivel de significación $\mathrm{p}<2 \% ; \mathrm{P}<1 \%$ : Número de puntos con un nivel de significación $\mathrm{p}<1 \% ; \mathrm{P}<0,5 \%$ : Número de puntos con un nivel de significación p<0,5\%; Área exc: Área de excavación; Área E/D: Área excavación/disco; Área A/D: Área anillo/disco; Vol exc: Volumen de excavación, Vol anillo: Volumen de anillo; Prof med exc: Profundidad media de excavación; Prof máx exc: Profundidad máxima de excavación; Var alt cont: Variación altura de contorno; Morf exc: Morfología de la excavación; Esp med CFNR: Espesor medio de la capa de fibras nerviosas de la retina; Sec CFNR; Sección capa de fibras nerviosas de la retina; Coc hor E/D: Cociente horizontal excavación/disco; Coc vert E/D: Cociente vertical excavación/disco; Máx elev cont: Máxima elevación del contorno; Máx dep cont: Máxima depresión del contorno; LMC TS: Línea de modulación del contorno temporal superior; LMC TI: Línea de modulación del contorno temporal inferior; Variab media: Variabilidad media; Alt referencia: Altura de referencia; FSM: Función discriminante lineal de Mikelberg FS; RB: Función discriminante lineal de Burk RO; Dx Moorfields: Diagnóstico del Análisis de regresión de Moorfields.

Las topografías papilares se realizaron bajo midriasis pupilar con colirio de tropicamida al $1 \%$ (Alcon Laboratorioes Inc, Fort Worth, TX, EEUU). El HRT utiliza un láser diodo rojo de $670 \mathrm{~nm}$ que recorre la retina iluminando cada punto en un corto espacio de tiempo y la luz reflejada por la retina es almacenada. Al repetir el proceso en toda la zona mediante barridos horizontales sucesivos, se logra construir una imagen de la superficie explorada. El aparato realiza automáticamente de 16 a 64 planos equidistantes. El área analizada corresponde a $15^{\circ} \mathrm{de}$ campo transversal y una profundidad de $0,5 \mathrm{a} 4 \mathrm{~mm}$, con una resolución transversal de 10 micras/píxel. Una vez obtenida la imagen bidimensional un explorador experimentado debe delimitar manualmente el contorno del disco óptico, tras lo cuál, el aparato establece de forma automática un plano de referencia para el cálculo de los parámetros topográficos. 
Tabla VI. Correlaciones entre los índices de la perimetría, el número de puntos alterados por cuadrantes y el número de puntos alterados por niveles de probabilidad con los parámetros de la CNO evaluados con el HRT en el grupo de glaucomas. Las correlaciones significativas $(p<0,05)$ están resaltadas en fondo gris

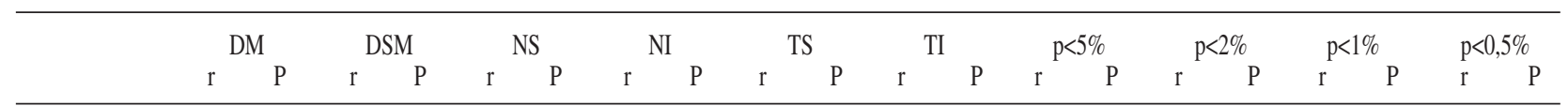

\begin{tabular}{llllllllllllllllllllllll}
\hline Área disco & $-0,103$ & 0,383 & 0,038 & 0,746 & $-0,011$ & 0,930 & $-0,047$ & 0,711 & 0,176 & 0,164 & 0,194 & 0,125 & 0,101 & 0,426 & 0,065 & 0,611 & 0,069 & 0,586 & 0,032 & 0,803
\end{tabular}

$\begin{array}{lllllllllllllllllllllll}\text { Área excav. } & -0,385 & 0,001 & 0,326 & 0,005 & 0,260 & 0,038 & 0,097 & 0,445 & 0,229 & 0,068 & 0,249 & 0,047 & 0,286 & 0,022 & 0,279 & 0,026 & 0,317 & 0,011 & 0,307 & 0,014\end{array}$

$\begin{array}{llllllllllllllllllllllll}\text { Área anillo } & 0,432 & 0,000 & -0,444 & 0,000 & -0,399 & 0,001 & -0,198 & 0,117 & -0,102 & 0,423 & -0,189 & 0,134 & -0,305 & 0,014 & -0,331 & 0,008 & -0,388 & 0,002 & -0,422 & 0,001\end{array}$

$\begin{array}{lllllllllllllllllllllllll}\text { Coc E/D } & -0,479 & 0,000 & 0,440 & 0,000 & 0,392 & 0,001 & 0,195 & 0,123 & 0,207 & 0,101 & 0,270 & 0,031 & 0,365 & 0,003 & 0,373 & 0,002 & 0,421 & 0,001 & 0,428 & 0,000\end{array}$

$\begin{array}{lllllllllllllllllllllll}\mathrm{Coc} A / \mathrm{D} & 0,479 & 0,000 & -0,440 & 0,000 & -0,392 & 0,001 & -0,195 & 0,123 & -0,207 & 0,101 & -0,270 & 0,031 & -0,365 & 0,003 & -0,373 & 0,002 & -0,421 & 0,001 & -0,428 & 0,000\end{array}$

$\begin{array}{lllllllllllllllllllllll}\text { Volumen exc } & -0,270 & 0,020 & 0,314 & 0,007 & 0,275 & 0,028 & 0,052 & 0,681 & 0,110 & 0,385 & 0,060 & 0,637 & 0,193 & 0,126 & 0,194 & 0,124 & 0,243 & 0,053 & 0,240 & 0,056\end{array}$

$\begin{array}{llllllllllllllllllllllllll}\text { Volumen anillo } & 0,301 & 0,009 & -0,287 & 0,013 & -0,297 & 0,017 & -0,091 & 0,473 & -0,087 & 0,494 & -0,095 & 0,454 & -0,183 & 0,147 & -0,204 & 0,107 & -0,235 & 0,062 & -0,247 & 0,049\end{array}$

$\begin{array}{llllllllllllllllllllll}\text { Prf med exc } & -0,175 & 0,135 & 0,248 & 0,033 & 0,220 & 0,080 & 0,020 & 0,872 & -0,044 & 0,731 & 0,000 & 0,998 & 0,092 & 0,472 & 0,078 & 0,541 & 0,123 & 0,334 & 0,127 & 0,318\end{array}$

$\begin{array}{lllllllllllllllllllll}\text { Prf máx exc } & -0,149 & 0,205 & 0,169 & 0,149 & 0,148 & 0,243 & 0,002 & 0,988 & 0,049 & 0,702 & 0,080 & 0,528 & 0,102 & 0,423 & 0,110 & 0,386 & 0,130 & 0,305 & 0,113 & 0,374\end{array}$

$\begin{array}{llllllllllllllllllllllll}\text { Var alt cnt } & -0,129 & 0,275 & 0,222 & 0,057 & 0,164 & 0,195 & -0,047 & 0,713 & 0,211 & 0,095 & 0,146 & 0,249 & 0,141 & 0,266 & 0,187 & 0,140 & 0,215 & 0,087 & 0,205 & 0,104\end{array}$

$\begin{array}{lllllllllllllllllllllll}\text { Morf excav. } & -0,298 & 0,010 & 0,295 & 0,011 & 0,227 & 0,072 & 0,131 & 0,302 & 0,007 & 0,958 & 0,093 & 0,466 & 0,179 & 0,156 & 0,148 & 0,243 & 0,195 & 0,122 & 0,218 & 0,083\end{array}$

$\begin{array}{llllllllllllllllllllllll}\text { Esp med CFNR } & 0,382 & 0,001 & -0,291 & 0,012 & -0,305 & 0,014 & -0,231 & 0,066 & -0,330 & 0,008 & -0,301 & 0,016 & -0,385 & 0,002 & -0,422 & 0,001 & -0,428 & 0,000 & -0,405 & 0,001\end{array}$ $\begin{array}{lllllllllllllllllllllllll}\text { Sección CFNR } & 0,355 & 0,002 & -0,277 & 0,017 & -0,290 & 0,020 & -0,222 & 0,078 & -0,319 & 0,010 & -0,270 & 0,031 & -0,363 & 0,003 & -0,404 & 0,001 & -0,409 & 0,001 & -0,389 & 0,001\end{array}$ $\begin{array}{lllllllllllllllllllllll}\text { Coc E/D hor } & -0,299 & 0,010 & 0,256 & 0,028 & 0,250 & 0,046 & 0,056 & 0,663 & 0,152 & 0,232 & 0,232 & 0,065 & 0,201 & 0,112 & 0,229 & 0,069 & 0,245 & 0,051 & 0,233 & 0,063\end{array}$ $\begin{array}{lrlllllllllllllllllll}\text { Coc E/D vert } & -0,442 & 0,000 & 0,371 & 0,001 & 0,366 & 0,003 & 0,173 & 0,171 & 0,243 & 0,053 & 0,314 & 0,012 & 0,005 & 0,375 & 0,002 & 0,405 & 0,001 & 0,396 & 0,001\end{array}$ $\begin{array}{llllllllllllllllllllll}\text { Máx elev cont } & -0,059 & 0,619 & -0,078 & 0,507 & -0,012 & 0,928 & -0,046 & 0,721 & -0,026 & 0,841 & -0,086 & 0,498 & -0,066 & 0,606 & -0,044 & 0,728 & -0,013 & 0,917 & -0,002 & 0,988\end{array}$ $\begin{array}{lllllllllllllllllllllll}\text { Máx dep cont } & -0,173 & 0,142 & 0,193 & 0,100 & 0,172 & 0,175 & -0,079 & 0,533 & 0,214 & 0,089 & 0,106 & 0,403 & 0,113 & 0,372 & 0,176 & 0,163 & 0,227 & 0,071 & 0,223 & 0,076\end{array}$ $\begin{array}{lllllllllllllllllllllllllll}\text { LMC TS } & 0,356 & 0,002 & -0,084 & 0,478 & -0,201 & 0,111 & -0,465 & 0,000 & -0,139 & 0,275 & -0,318 & 0,011 & -0,393 & 0,001 & -0,396 & 0,001 & -0,386 & 0,002 & -0,363 & 0,003\end{array}$ $\begin{array}{llllllllllllllllllllll}\text { LMC TI } & 0,240 & 0,040 & -0,391 & 0,001 & -0,363 & 0,003 & -0,023 & 0,855 & -0,270 & 0,031 & -0,032 & 0,804 & -0,209 & 0,098 & -0,289 & 0,021 & -0,388 & 0,006 & -0,352 & 0,004\end{array}$ $\begin{array}{llllllllllllllllllllllll}\text { Variab med } & -0,188 & 0,109 & 0,141 & 0,230 & 0,079 & 0,533 & 0,222 & 0,078 & 0,159 & 0,210 & 0,320 & 0,010 & 0,274 & 0,028 & 0,276 & 0,027 & 0,266 & 0,034 & 0,248 & 0,048\end{array}$ $\begin{array}{lllllllllllllllllllllll}\text { Altura ref. } & 0,149 & 0,206 & -0,154 & 0,191 & -0,182 & 0,149 & -0,304 & 0,014 & -0,202 & 0,109 & -0,206 & 0,102 & -0,335 & 0,007 & -0,300 & 0,016 & -0,249 & 0,047 & -0,218 & 0,083\end{array}$ $\begin{array}{lllllllllllllllllllllll}\text { FDL FSM } & 0,346 & 0,003 & -0,425 & 0,000 & -0,361 & 0,003 & -0,043 & 0,738 & -0,187 & 0,139 & -0,180 & 0,155 & -0,242 & 0,054 & -0,284 & 0,023 & -0,345 & 0,005 & -0,356 & 0,004\end{array}$ $\begin{array}{lllllllllllllllllllllllll}\text { FDL RB } & 0,426 & 0,000 & -0,412 & 0,000 & -0,400 & 0,001 & -0,276 & 0,028 & -0,193 & 0,126 & -0,228 & 0,070 & -0,335 & 0,004 & -0,423 & 0,000 & -0,472 & 0,000 & -0,480 & 0,000\end{array}$ $\begin{array}{lllllllllllllllllllll}\text { Dx Moorfields } & -0,310 & 0,007 & 0,273 & 0,018 & 0,195 & 0,123 & 0,054 & 0,672 & 0,162 & 0,202 & 0,200 & 0,113 & 0,187 & 0,139 & 0,245 & 0,051 & 0,297 & 0,017 & 0,293 & 0,019\end{array}$

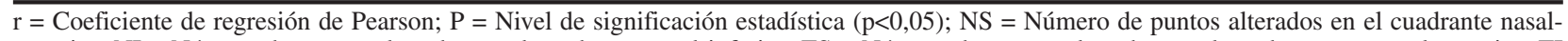
superior; NI = Número de puntos alterados en el cuadrante nasal-inferior; TS = Número de puntos alterados en el cuadrante temporal-superior; TI $=$ Número de puntos alterados en el cuadrante temporal-inferior; $\mathrm{P}<5 \%=$ Número de puntos con un nivel de significación p $<5 \% ; \mathrm{P}<2 \%=\mathrm{Número}$ de puntos con un nivel de significación $\mathrm{p}<2 \% ; \mathrm{P}<1 \%=$ Número de puntos con un nivel de significación $\mathrm{p}<1 \%$; $\mathrm{P}<0,5 \%=$ : Número de puntos con un nivel de significación p $<0,5 \%$; Área exc = Área de excavación; Área E/D = Área excavación/disco; Área A/D = Área anillo/disco; Vol exc = Volumen de excavación, Vol anillo = Volumen de anillo; Prof med exc = Profundidad media de excavación; Prof máx exc = Profundidad máxima de excavación; Var alt cont $=$ Variación altura de contorno; Morf exc = Morfología de la excavación; Esp med CFNR = Espesor medio de la capa de fibras nerviosas de la retina; Sec CFNR; Sección capa de fibras nerviosas de la retina; Coc hor E/D = Cociente horizontal excavación/disco; Coc vert E/D = Cociente vertical excavación/disco; Máx elev cont = Máxima elevación del contorno; Máx dep cont = Máxima depresión del contorno; LMC TS = Línea de modulación del contorno temporal superior; LMC TI = Línea de modulación del contorno temporal inferior; Variab media = Variabilidad media; Alt referencia = Altura de referencia; FSM = Función discriminante lineal de Mikelberg FS; RB = Función discriminante lineal de Burk RO; Dx Moorfields = Diagnóstico del Análisis de regresión de Moorfields.

\section{Clasificación en grupos}

- Normales (87): PIO $<21 \mathrm{mmHg}$, papila normal, y PA normal.

- Hipertensos oculares (HTO; 192): PIO $\geq 21$ $\mathrm{mmHg}$, papila normal, y PA normal.

- Sospechosos de glaucoma (70): PIO $\geq 21$ $\mathrm{mmHg}$, papila de morfología glaucomatosa, y PA normal.

- Glaucomas (74): PIO $\geq 21 \mathrm{mmHg}$, papila alterada, y PA alterada.

\section{Análisis estadístico}

Los análisis estadísticos se realizaron con el programa SPSS (versión 15.0; SPSS Inc., Chicago, IL, EEUU) y MedCalc (versión 9.3.2.0 MedCalc Software, Mariakerke, Bélgica).

Todas las variables estudiadas seguían una distribución normal tal y como se comprobó mediante el test de Kolmogorov-Smirnov (K-S de 1 muestra). Para comparar las variables cuantitativas entre los diferentes grupos se utilizó el análisis de la varian- 


\begin{tabular}{|l|l|l|l|l|l|l|l|l|}
\hline & & $-0,214$ & $-0,035$ & 0,005 & $-0,006$ & & \\
\hline & $-0,111$ & 0,007 & 0,020 & $-0,179$ & 0,010 & $-0,069$ & \\
\hline & $-0,112$ & $-0,230$ & $-0,045$ & 0,001 & $-0,108$ & $-0,013$ & 0,070 & $-0,137$ \\
\hline$-0,305$ & $-0,056$ & $-0,031$ & 0,180 & 0,100 & $-0,028$ & $-0,072$ & & $-0,046$ \\
\hline$-0,147$ & $-0,104$ & $-0,104$ & $-0,108$ & $-0,096$ & $-0,060$ & $-0,075$ & & $-0,011$ \\
\hline & $-0,196$ & $-0,035$ & 0,093 & $-0,157$ & 0,050 & 0,040 & 0,093 & $-0,087$ \\
\hline & & 0,001 & 0,049 & 0,015 & $-0,082$ & $-0,008$ & $-0,010$ & \\
\hline & & $-0,110$ & $-0,078$ & $-0,044$ & 0,039 & & \\
\hline
\end{tabular}

Grupo normal

\begin{tabular}{|l|l|l|l|l|l|l|l|l|}
\hline & & & 0,138 & $\mathbf{0 , 3 1 1}$ & 0,254 & 0,223 & & \\
\hline & $-0,021$ & $\mathbf{0 , 2 7 6}$ & 0,234 & 0,138 & $\mathbf{0 , 1 3 7}$ & $\mathbf{0 , 2 5 6}$ & \\
\hline & 0,091 & 0,239 & $\mathbf{0 , 2 8 1}$ & 0,217 & $\mathbf{0 , 3 6 7}$ & 0,198 & 0,228 & $\mathbf{0 , 3 0 0}$ \\
\hline $\mathbf{0 , 2 6 8}$ & 0,138 & 0,236 & 0,104 & 0,151 & 0,076 & $\mathbf{0 , 3 5 4}$ & & 0,182 \\
\hline $\mathbf{0 , 1 5 4}$ & $\mathbf{0 , 2 8 3}$ & $\mathbf{0 , 2 7 8}$ & 0,206 & 0,198 & 0,073 & 0,236 & & 0,144 \\
\hline & $\mathbf{0 , 3 6 4}$ & 0,054 & $\mathbf{0 , 2 5 8}$ & $\mathbf{0 , 2 8 3}$ & $\mathbf{0 , 2 8 6}$ & $\mathbf{0 , 2 7 1}$ & 0,130 & 0,086 \\
\hline & & 0,158 & 0,216 & 0,253 & 0,177 & $\mathbf{0 , 1 2 6}$ & 0,082 & \\
\hline & & & $\mathbf{0 , 1 4 0}$ & $\mathbf{0 , 3 8 3}$ & $\mathbf{0 , 3 1 6}$ & $\mathbf{0 , 2 6 6}$ & & \\
\hline
\end{tabular}

Grupo glaucoma pre-perimétrico

\begin{tabular}{|l|l|l|l|l|l|l|l|l|}
\hline & & & $-0,004$ & 0,060 & 0,001 & 0,031 & & \\
\hline & 0,006 & 0,034 & 0,046 & 0,101 & $\mathbf{0 , 1 6 3}$ & 0,042 & \\
\hline & 0,077 & 0,059 & $-0,003$ & 0,035 & $-0,021$ & $-0,035$ & 0,008 & 0,034 \\
\hline 0,024 & 0,120 & 0,008 & 0,047 & $-0,034$ & 0,115 & $-0,105$ & & $-0,014$ \\
\hline 0,083 & 0,139 & 0,093 & 0,013 & 0,010 & 0,034 & $-0,085$ & & $-0,067$ \\
\hline & 0,070 & 0,001 & 0,078 & $-0,037$ & 0,046 & 0,017 & $-0,041$ & $-0,011$ \\
\hline & & $-0,003$ & 0,001 & 0,048 & 0,006 & 0,005 & 0,087 & \\
\hline & & & $\mathbf{0 , 1 7 6}$ & 0,090 & 0,083 & 0,125 & & \\
\hline
\end{tabular}

\section{Grupo HTO}

\begin{tabular}{|l|l|l|l|l|l|l|l|l|}
\hline & & $\mathbf{0 , 4 4 6}$ & $\mathbf{0 , 3 0 1}$ & $\mathbf{0 , 3 0 4}$ & $\mathbf{0 , 2 2 7}$ & & \\
\hline & $\mathbf{0 , 4 1 3}$ & $\mathbf{0 , 3 7 3}$ & $\mathbf{0 , 3 9 4}$ & $\mathbf{0 , 4 1 4}$ & $\mathbf{0 , 3 3 8}$ & $\mathbf{0 , 3 0 7}$ & \\
\hline & $\mathbf{0 , 3 6 8}$ & $\mathbf{0 , 4 8 3}$ & $\mathbf{0 , 4 1 5}$ & $\mathbf{0 , 4 9 8}$ & $\mathbf{0 , 3 6 0}$ & $\mathbf{0 , 3 9 3}$ & $\mathbf{0 , 2 7 0}$ & 0,098 \\
\hline $\mathbf{0 , 4 4 4}$ & $\mathbf{0 , 3 8 0}$ & $\mathbf{0 , 4 4 1}$ & $\mathbf{0 , 3 0 2}$ & $\mathbf{0 , 3 6 1}$ & $\mathbf{0 , 3 8 9}$ & $\mathbf{0 , 4 4 2}$ & & 0,050 \\
\hline $\mathbf{0 , 3 5 6}$ & $\mathbf{0 , 3 7 6}$ & $\mathbf{0 , 3 2 5}$ & $\mathbf{0 , 3 0 2}$ & $\mathbf{0 , 2 6 4}$ & $\mathbf{0 , 3 2 9}$ & $\mathbf{0 , 3 0 1}$ & & 0,080 \\
\hline & 0,214 & $\mathbf{0 , 4 0 9}$ & $\mathbf{0 , 3 0 1}$ & $\mathbf{0 , 3 6 8}$ & $\mathbf{0 , 3 3 9}$ & $\mathbf{0 , 3 5 6}$ & $\mathbf{0 , 2 7 0}$ & 0,112 \\
\hline & & $\mathbf{0 , 3 7 8}$ & $\mathbf{0 , 3 1 0}$ & $\mathbf{0 , 3 3 8}$ & 0,175 & 0,219 & 0,164 & \\
\hline & & & 0,170 & $\mathbf{0 , 2 4 7}$ & 0,174 & $\mathbf{0 , 2 9 4}$ & & \\
\hline
\end{tabular}

\section{Grupo glaucomas}

Fig. 1: Representación gráfica de los coeficientes de correlación de Pearson obtenidos entre el área del anillo neurorretiniano y los 52 puntos del campo visual en los 4 grupos de estudio. Las correlaciones significativas están resaltadas en negrita.

za (ANOVA) con la corrección de Bonferroni para múltiples comparaciones. La fuerza de asociación entre las variables cuantitativas se determinó con el coeficiente de correlación de Pearson. Si este mostraba valores inferiores a 0,4 las correlaciones se consideraron débiles, entre 0,4 y 0,7 moderadas y aquellas con valores superiores a 0,7 fueron consideradas fuertes.

\section{RESULTADOS}

Las características clínicas de la población de estudio se muestran en la tabla I. No se encontraron diferencias en la edad de los diferentes grupos diagnósticos. El grupo de glaucomas presentó una mejor agudeza visual corregida menor que los otros tres, la PIO fue menor en el grupo normal que en los demás y los HTO presentaron mayores valores paquimétricos. En cuanto a la relación excavación/disco vertical, en los normales e HTO fue diferente de los sospechosos de glaucoma y glaucomas. La DM fue más negativa y la DSM más positiva en el grupo de glaucomas que en el resto. El grado de daño en la perimetría del grupo de glaucomas fue leve o moderado (10). Las diferencias entre los grupos de los valores de PIO, cociente excavación/disco e índices perimétricos se justifi- 


\begin{tabular}{|l|l|l|l|l|l|l|l|l|}
\hline & & & 0,174 & 0,206 & 0,083 & $-0,051$ & & \\
\hline & & 0,146 & 0,029 & $\mathbf{0 , 2 5 8}$ & 0,159 & 0,032 & 0,043 & \\
\hline & 0,216 & 0,219 & 0,096 & 0,092 & $-0,040$ & $-0,046$ & $-0,006$ & 0,144 \\
\hline $\mathbf{0 , 2 8 2}$ & 0,161 & 0,151 & 0,035 & $-0,096$ & $-0,035$ & $-0,122$ & & $-0,034$ \\
\hline 0,217 & 0,301 & 0,232 & $-0,001$ & 0,101 & 0,066 & 0,021 & & $-0,054$ \\
\hline & 0,301 & 0,158 & $-0,136$ & 0,037 & $-0,067$ & $-0,128$ & $-0,145$ & 0,020 \\
\hline & & 0,155 & 0,142 & 0,161 & 0,113 & 0,027 & 0,003 & \\
\hline & & & 0,087 & 0,209 & 0,092 & 0,003 & & \\
\hline
\end{tabular}

Grupo normal

\begin{tabular}{|l|l|l|l|l|l|l|l|l|}
\hline & & $-0,043$ & $-0,142$ & $-0,106$ & $-0,107$ & & \\
\hline & 0,083 & $-0,079$ & $-0,057$ & $-0,009$ & $-0,054$ & $-0,083$ & \\
\hline & $-0,034$ & $-0,216$ & $-0,290$ & $-0,268$ & $-0,281$ & $-0,154$ & $-0,148$ & $-0,221$ \\
\hline$-0,170$ & $-0,116$ & $-0,260$ & $-0,087$ & $-0,310$ & $-0,228$ & $-0,396$ & & $-0,281$ \\
\hline$-0,063$ & $-0,185$ & $-0,164$ & 0,212 & $-0,268$ & 0,226 & $-0,330$ & & $-0,238$ \\
\hline & $-0,221$ & $-0,157$ & $-0,196$ & $-0,284$ & $-0,248$ & $-0,367$ & $-0,250$ & $-0,136$ \\
\hline & 0,058 & $-0,174$ & $-0,238$ & $-0,059$ & 0,003 & $-0,068$ & \\
\hline & & & 0,021 & $-0,245$ & $-0,130$ & $-0,284$ & & \\
\hline
\end{tabular}

Grupo glaucoma pre-perimétrico

\begin{tabular}{|l|l|l|l|l|l|l|l|l|}
\hline & & & 0,122 & 0,140 & 0,127 & 0,107 & & \\
\hline & 0,127 & $\mathbf{0 , 1 8 9}$ & $\mathbf{0 , 1 6 7}$ & 0,057 & 0,064 & 0,117 & \\
\hline & $\mathbf{0 , 2 0 8}$ & $\mathbf{0 , 2 1 4}$ & $\mathbf{0 , 1 9 9}$ & 0,099 & 0,083 & 0,137 & $\mathbf{0 , 1 9 6}$ & $\mathbf{0 , 1 7 7}$ \\
\hline 0,034 & $\mathbf{0 , 1 2 5}$ & 0,049 & $\mathbf{0 , 1 5 5}$ & 0,057 & 0,037 & 0,076 & & 0,000 \\
\hline 0,049 & 0,028 & $\mathbf{0 , 1 6 9}$ & $\mathbf{0 , 0 6 9}$ & 0,048 & $-0,106$ & 0,089 & & 0,077 \\
\hline & 0,111 & 0,125 & $\mathbf{0 , 1 4 9}$ & 0,064 & 0,068 & 0,091 & 0,137 & 0,123 \\
\hline & & 0,110 & 0,123 & $\mathbf{0 , 1 9 2}$ & $\mathbf{0 , 1 7 2}$ & $-0,060$ & 0,055 & \\
\hline & & & 0,053 & 0,016 & 0,036 & $\mathbf{0 , 1 6 7}$ & & \\
\hline
\end{tabular}

Grupo HTO

\begin{tabular}{|l|l|l|l|l|l|l|l|l|}
\hline & & $-0,389$ & $-0,323$ & $-0,330$ & $-0,285$ & & \\
\hline & $-0,363$ & $-0,332$ & $-0,403$ & $-0,442$ & $-0,384$ & $-0,368$ & \\
\hline & $-0,367$ & $-0,466$ & $-0,387$ & $-0,477$ & $-0,385$ & $-0,351$ & $-0,335$ & $-0,223$ \\
\hline$-0,448$ & $-0,366$ & $-0,412$ & $-0,268$ & $-0,311$ & $-0,365$ & $-0,449$ & & $-0,208$ \\
\hline$-0,374$ & $-0,372$ & $-0,316$ & $-0,320$ & $-0,290$ & $-0,295$ & $-0,345$ & & $-0,268$ \\
\hline & $-0,190$ & $-0,404$ & $-0,277$ & $-0,337$ & $-0,387$ & $-0,312$ & $-0,343$ & $-0,274$ \\
\hline & & $-0,352$ & $-0,327$ & $-0,409$ & $-0,299$ & $-0,342$ & $-0,280$ & \\
\hline & & & $-0,362$ & $-0,324$ & $-0,290$ & $-0,335$ & & \\
\hline
\end{tabular}

Grupo glaucomas

Fig. 2: Representación gráfica de los coeficientes de correlación de Pearson obtenidos entre el cociente excavación/disco y los 52 puntos del campo visual en los 4 grupos de estudio. Las correlaciones significativas están resaltadas en negrita.

can por los criterios de clasificación empleados para establecer los grupos. En la tabla II se comparan los parámetros del HRT entre los diferentes grupos. La mayoría de ellos mostraron diferencias entre los grupos de normales e hipertensos, y los grupos de sospechosos y glaucomatosos.

Las correlaciones de los parámetros del HRT II con los índices perimétricos (tablas III-VI), fueron escasas y de carácter débil en los sujetos normales, HTO y sospechosos de glaucoma. Sin embargo, en el grupo de glaucomas aumentó el número y la fuerza de las mismas, destacando las establecidas entre los cocientes excavación/disco y anillo/disco con la $\mathrm{DM}(\mathrm{r}=0,479)$ y entre el área de anillo con la DSM $(\mathrm{r}=-0,444)$. Además, en este grupo también presentaron correlaciones moderadas (en torno a 0,4) el espesor y la sección transversal de la capa de fibras nerviosas de la retina (CFNR), el cociente excavación/disco vertical y las funciones discriminantes FSM (11) y RB (12). La DM presentó correlaciones más fuertes que la DSM en todos los grupos, salvo en el grupo normal. En los sujetos glaucomatosos además de la DM, el número de puntos alterados con $\mathrm{p}<1 \%$ y $\mathrm{p}<0,5 \%$ presentaron las correlaciones más fuertes con los parámetros del HRT II.

Al analizar las correlaciones establecidas entre las variables del HRT II y los valores umbral de 


\begin{tabular}{|l|l|l|l|l|l|l|l|l|}
\hline & & & 0,041 & 0,111 & $-0,122$ & 0,145 & & \\
\hline & $-0,231$ & $-0,134$ & $-0,058$ & $-0,065$ & 0,041 & 0,113 & \\
\hline & $-0,138$ & $-0,150$ & $-0,082$ & $-0,073$ & 0,049 & 0,073 & $-0,009$ & $-0,029$ \\
\hline$-0,064$ & $-0,131$ & $-0,131$ & 0,019 & 0,078 & 0,026 & 0,010 & & 0,078 \\
\hline 0,039 & $-0,216$ & $-0,206$ & 0,053 & $-0,264$ & $-0,064$ & $-0,112$ & & $-0,154$ \\
\hline & $-0,243$ & $-0,041$ & $-0,095$ & $-0,298$ & $-0,064$ & 0,108 & 0,048 & 0,022 \\
\hline & & $-0,081$ & $-0,055$ & $-0,031$ & $-0,141$ & $-0,279$ & 0,181 & \\
\hline & & & $-0,030$ & $-0,003$ & 0,035 & $-0,106$ & & \\
\hline
\end{tabular}

\section{Grupo normal}

\begin{tabular}{|l|l|l|l|l|l|l|l|l|}
\hline & & & $\mathbf{0 , 2 8 1}$ & $\mathbf{0 , 2 6 7}$ & 0,155 & $\mathbf{0 , 3 8 5}$ & & \\
\hline & & 0,237 & $\mathbf{0 , 3 0 0}$ & $\mathbf{0 , 2 7 2}$ & 0,216 & 0,277 & $\mathbf{0 , 2 8 7}$ & \\
\hline & 0,244 & $\mathbf{0 , 3 5 8}$ & $\mathbf{0 , 3 3 3}$ & $\mathbf{0 , 2 6 7}$ & $\mathbf{0 , 4 3 0}$ & 0,113 & $\mathbf{0 , 3 9 1}$ & $\mathbf{0 , 3 7 3}$ \\
\hline $\mathbf{0 , 3 6 4}$ & $\mathbf{0 , 3 3 6}$ & $\mathbf{0 , 3 9 4}$ & $\mathbf{0 , 2 8 0}$ & 0,224 & $\mathbf{0 , 2 9 2}$ & $\mathbf{0 , 4 2 4}$ & & $\mathbf{0 , 3 4 8}$ \\
\hline $\mathbf{0 , 2 8 5}$ & $\mathbf{0 , 3 7 8}$ & $\mathbf{0 , 4 2 2}$ & $\mathbf{0 , 3 9 5}$ & 0,163 & $\mathbf{0 , 2 7 5}$ & $\mathbf{0 , 3 0 6}$ & & $\mathbf{0 , 4 2 1}$ \\
\hline & $\mathbf{0 , 4 9 6}$ & $\mathbf{0 , 2 8 2}$ & 0,247 & $\mathbf{0 , 4 4 7}$ & $\mathbf{0 , 4 3 7}$ & $\mathbf{0 , 4 5 2}$ & $\mathbf{0 , 4 5 3}$ & $\mathbf{0 , 2 6 2}$ \\
\hline & & 0,147 & $\mathbf{0 , 2 8 3}$ & $\mathbf{0 , 3 7 7}$ & $\mathbf{0 , 2 5 7}$ & 0,249 & $\mathbf{0 , 3 6 4}$ & \\
\hline & & & 0,133 & $\mathbf{0 , 2 8 5}$ & $\mathbf{0 , 2 9 1}$ & $\mathbf{0 , 3 2 6}$ & & \\
\hline
\end{tabular}

Grupo glaucoma pre-perimétrico

\begin{tabular}{|l|l|l|l|l|l|l|l|l|}
\hline & & 0,069 & 0,087 & 0,016 & 0,003 & & \\
\hline & $-0,017$ & $-0,081$ & $-0,004$ & $-0,044$ & 0,024 & $-0,046$ & \\
\hline & $-0,015$ & $-0,093$ & $-0,093$ & $-0,075$ & $-0,027$ & 0,010 & $-0,024$ & $-0,051$ \\
\hline$-0,004$ & $-0,034$ & $-0,040$ & $-0,056$ & 0,034 & 0,140 & 0,026 & & $-0,051$ \\
\hline$-0,003$ & 0,009 & $-0,015$ & 0,015 & 0,091 & 0,072 & 0,008 & & $-0,057$ \\
\hline & $-0,019$ & 0,033 & $-0,032$ & $-0,046$ & $-0,017$ & $-0,006$ & $-0,124$ & $-0,077$ \\
\hline & & $-0,007$ & $-0,121$ & $-0,031$ & $-0,009$ & $-0,009$ & $-0,078$ & \\
\hline & & & $-0,012$ & 0,101 & $-0,047$ & 0,036 & & \\
\hline
\end{tabular}

\section{Grupo HTO}

\begin{tabular}{|l|l|l|l|l|l|l|l|l|}
\hline & & $\mathbf{0 , 3 2 1}$ & 0,244 & 0,246 & $\mathbf{0 , 2 5 2}$ & & \\
\hline & & $\mathbf{0 , 3 2 1}$ & $\mathbf{0 , 2 8 8}$ & 0,345 & $\mathbf{0 , 4 1 9}$ & $\mathbf{0 , 3 3 2}$ & $\mathbf{0 , 4 0 8}$ & \\
\hline & $\mathbf{0 , 3 1 5}$ & $\mathbf{0 , 4 3 4}$ & 0,241 & $\mathbf{0 , 3 1 2}$ & $\mathbf{0 , 3 0 0}$ & $\mathbf{0 , 2 8 6}$ & $\mathbf{0 , 3 3 4}$ & $\mathbf{0 , 3 6 3}$ \\
\hline $\mathbf{0 , 2 9 8}$ & $\mathbf{0 , 3 5 6}$ & $\mathbf{0 , 3 9 8}$ & 0,224 & $\mathbf{0 , 2 7 8}$ & $\mathbf{0 , 3 6 6}$ & $\mathbf{0 , 4 3 4}$ & & $\mathbf{0 , 4 0 4}$ \\
\hline $\mathbf{0 , 2 9 2}$ & $\mathbf{0 , 2 7 0}$ & 0,240 & 0,243 & $\mathbf{0 , 2 6 9}$ & $\mathbf{0 , 2 2 2}$ & $\mathbf{0 , 3 1 1}$ & & $\mathbf{0 , 1 5 4}$ \\
\hline & $\mathbf{0 , 1 8 7}$ & $\mathbf{0 , 3 0 1}$ & $\mathbf{0 , 2 6 5}$ & $\mathbf{0 , 2 7 8}$ & 0,193 & $\mathbf{0 , 2 3 4}$ & 0,243 & $\mathbf{0 , 0 8 7}$ \\
\hline & & $\mathbf{0 , 2 8 7}$ & 0,276 & 0,215 & 0,176 & $\mathbf{0 , 3 0 3}$ & 0,142 & \\
\hline & & & 0,125 & 0,222 & 0,208 & $\mathbf{0 , 3 1 7}$ & & \\
\hline
\end{tabular}

\section{Grupo glaucomas}

Fig. 3: Representación gráfica de los coeficientes de correlación de Pearson obtenidos entre el espesor medio de la capa de fibras nerviosas de la retina y los 52 puntos del campo visual en los 4 grupos de estudio. Las correlaciones significativas están resaltadas en negrita.

los 52 puntos del campo visual (figs. 1-5), se observó la misma tendencia. Las figuras 1-5 incluyen los parámetros del HRT II que destacaron en cuanto al número y fuerza de las correlaciones; área de anillo, cociente excavación/disco, espesor medio de la CFNR, líneas de modulación del contorno temporal-superior y temporal-inferior. Los HTO resultaron ser los que menor número y fuerza de correlaciones presentaron, por debajo incluso de los sujetos normales donde el coeficiente de correlación de Pearson alcanzó en alguna ocasión valores de 0,3. En los sujetos sospechosos y con glaucoma, se alcanzaron valores de 0,5 , aunque fueron más numerosas en los segundos. En los sospechosos de glaucoma destacó la línea de modulación de contorno temporalinferior, mientras que en los glaucomatosos fue la línea de modulación de contorno temporal-superior. En general, en todos los grupos se observaron mayor número de correlaciones estadísticamente significativas en la mitad superior del campo visual.

\section{DISCUSIÓN}

La diferente naturaleza de los parámetros estudiados hace que las correlaciones obtenidas sean de 


\begin{tabular}{|l|l|l|l|l|l|l|l|l|}
\hline & & 0,010 & 0,099 & $-0,104$ & $-0,007$ & & \\
\hline & & $-0,210$ & $-0,138$ & $-0,052$ & $-0,043$ & $-0,004$ & $-0,060$ & \\
\hline & $-0,033$ & $-0,101$ & $-0,040$ & 0,039 & 0,026 & $-0,025$ & 0,025 & $-0,024$ \\
\hline$-0,013$ & $-0,053$ & $-0,010$ & 0,121 & 0,048 & 0,029 & $-0,078$ & & 0,095 \\
\hline 0,115 & $-0,032$ & $-0,087$ & 0,016 & $-0,208$ & $-0,076$ & $-0,117$ & & $-0,173$ \\
\hline & $-0,150$ & $-0,029$ & 0,000 & $-0,279$ & $-0,033$ & $-0,067$ & $-0,066$ & $-0,018$ \\
\hline & & $-0,007$ & $-0,041$ & 00,049 & $-0,040$ & $-0,222$ & 0,069 & \\
\hline & & & $-0,091$ & 0,034 & 0,085 & $-0,065$ & & \\
\hline
\end{tabular}

\section{Grupo normal}

\begin{tabular}{|l|l|l|l|l|l|l|l|l|}
\hline & & $\mathbf{0 , 3 6 4}$ & $\mathbf{0 , 2 8 6}$ & $\mathbf{0 , 3 0 8}$ & $\mathbf{0 , 4 3 6}$ & & \\
\hline & & $\mathbf{0 , 2 5 8}$ & $\mathbf{0 , 3 2 4}$ & $\mathbf{0 , 2 8 7}$ & $\mathbf{0 , 3 3 7}$ & $\mathbf{0 , 3 0 9}$ & $\mathbf{0 , 3 9 9}$ & \\
\hline & $\mathbf{0 , 2 2 8}$ & $\mathbf{0 , 4 4 0}$ & $\mathbf{0 , 4 7 7}$ & $\mathbf{0 , 3 2 2}$ & $\mathbf{0 , 4 4 0}$ & $\mathbf{0 , 3 1 6}$ & $\mathbf{0 , 5 0 3}$ & $\mathbf{0 , 4 6 1}$ \\
\hline $\mathbf{0 , 3 6 8}$ & $\mathbf{0 , 3 4 5}$ & $\mathbf{0 , 4 8 1}$ & $\mathbf{0 , 3 6 3}$ & $-0,160$ & $-0,055$ & $-0,114$ & & $\mathbf{0 , 0 0 8}$ \\
\hline 0,207 & 0,172 & 0,120 & 0,018 & $-0,242$ & $-0,096$ & $-0,173$ & & $\mathbf{0 , 1 1 4}$ \\
\hline & 0,187 & $-0,157$ & $-0,092$ & 0,146 & 0,001 & $\mathbf{0 , 1 0 6}$ & $-0,052$ & $-0,011$ \\
\hline & & 0,064 & $-0,017$ & 0,054 & $-0,130$ & 0,170 & 0,151 & \\
\hline & & & $-0,010$ & $-0,075$ & $-0,078$ & $-0,043$ & & \\
\hline
\end{tabular}

Grupo glaucoma pre-perimétrico

\begin{tabular}{|l|l|l|l|l|l|l|l|l|}
\hline & & & 0,018 & 0,044 & $-0,045$ & $-0,036$ & & \\
\hline & & $-0,046$ & $-0,053$ & 0,047 & $-0,038$ & 0,023 & $-0,043$ & \\
\hline & 0,023 & $-0,057$ & $-0,103$ & $-0,061$ & $-0,022$ & $-0,034$ & 0,031 & 0,020 \\
\hline 0,032 & $-0,009$ & $-0,020$ & $-0,084$ & 0,036 & 0,079 & 0,048 & & $-0,003$ \\
\hline 0,045 & 0,018 & 0,001 & 0,028 & 0,034 & $-0,002$ & $-0,078$ & & $-0,042$ \\
\hline & $-0,002$ & 0,056 & $-0,080$ & $-0,052$ & $-0,015$ & $-0,057$ & $-0,110$ & $-0,050$ \\
\hline & & $-0,077$ & $-0,087$ & 0,044 & 0,006 & $-0,044$ & $-0,131$ & \\
\hline & & & 0,030 & 0,118 & 0,068 & 0,017 & & \\
\hline
\end{tabular}

Grupo HTO

\begin{tabular}{|l|l|l|l|l|l|l|l|l|}
\hline & & & $\mathbf{0 , 2 4 8}$ & 0,110 & 0,202 & 0,120 & & \\
\hline & $\mathbf{0 , 2 6 4}$ & $\mathbf{0 , 2 8 8}$ & 0,210 & $\mathbf{0 , 2 8 4}$ & 0,229 & $\mathbf{0 , 2 6 7}$ & \\
\hline $\mathbf{0 , 3 0 4}$ & $\mathbf{0 , 3 3 0}$ & $\mathbf{0 , 3 4 2}$ & 0,193 & 0,021 & 0,199 & $\mathbf{0 , 3 3 6}$ & & 0,110 \\
\hline $\mathbf{0 , 5 1 1}$ & $\mathbf{0 , 5 2 3}$ & $\mathbf{0 , 5 2 4}$ & $\mathbf{0 , 4 7 0}$ & $\mathbf{0 , 3 7 9}$ & $\mathbf{0 , 2 7 2}$ & $\mathbf{0 , 3 0 9}$ & & 0,051 \\
\hline & $\mathbf{0 , 4 6 9}$ & $\mathbf{0 , 4 8 2}$ & $\mathbf{0 , 5 3 3}$ & $\mathbf{0 , 5 4 4}$ & 0,212 & $\mathbf{0 , 4 0 1}$ & $\mathbf{0 , 3 4 5}$ & 0,121 \\
\hline & & $\mathbf{0 , 5 0 6}$ & $\mathbf{0 , 5 3 9}$ & $\mathbf{0 , 4 3 1}$ & $\mathbf{0 , 3 5 8}$ & $\mathbf{0 , 3 9 7}$ & 0,118 & \\
\hline & & & $\mathbf{0 , 2 5 2}$ & $\mathbf{0 , 4 0 0}$ & $\mathbf{0 , 4 1 4}$ & $\mathbf{0 , 3 8 0}$ & & \\
\hline
\end{tabular}

Grupo glaucomas

Fig. 4: Representación gráfica de los coeficientes de correlación de Pearson obtenidos entre la línea de modulación de contorno temporal-superior y los 52 puntos del campo visual en los 4 grupos de estudio. Las correlaciones significativas están resaltadas en negrita.

carácter débil, llegando a alcanzar en el mejor de los casos, valores del coeficiente de correlación de Pearson cercanos al 0,5. Entre los parámetros funcionales con mayor número de correlaciones significativas destaca la DM. En buena medida este resultado viene condicionado por las características de la población seleccionada, ya que en presencia de medios ópticos transparentes, la DM puede reflejar el componente focal en ausencia de pérdida difusa. Otras variables perimétricas que destacaron por su asociación con los parámetros papilares fueron el número de puntos alterados con $\mathrm{p}<1 \%$ y $\mathrm{p}<0,5 \%$.

En nuestro estudio, al igual que en trabajos previos (13), las correlaciones más fuertes se observa- ron en el grupo de glaucomas. Iester y colaboradores (14) no encontraron correlaciones significativas en el grupo de sujetos normales, y en los HTO, sólo detectaron asociaciones entre el área y el volumen del anillo neurorretiniano con la DSM. En su estudio, en el grupo de glaucomas (de alta y baja tensión), las asociaciones fueron más numerosas para la DM y la DSM, y el mejor indicador de cambio en estos índices fue el área del anillo neurorretiniano, y en segundo lugar la morfología de la excavación (CSM). Brigatti y colaboradores (15) sólo observaron un coeficiente de Pearson significativo entre la morfología de la excavación y los índices del campo visual en los sujetos con glaucoma, al igual que Uchida (16). Lan y colaboradores (17) sugirieron 


\begin{tabular}{|l|l|l|l|l|l|l|l|l|}
\hline & & & 0,122 & $\mathbf{0 , 2 7 2}$ & $-0,126$ & $\mathbf{0 , 2 3 5}$ & & \\
\hline & & $-0,072$ & 0,005 & 0,084 & 0,089 & 0,206 & 0,198 & \\
\hline & $-0,159$ & 0,036 & $-0,013$ & 0,094 & 0,208 & 0,141 & 0,067 & 0,049 \\
\hline 0,017 & $-0,051$ & $-0,040$ & $-0,023$ & 0,047 & 0,194 & 0,092 & & 0,123 \\
\hline 0,063 & $-0,122$ & $-0,030$ & 0,144 & $-0,147$ & 0,125 & 0,075 & & 0,028 \\
\hline & $-0,070$ & 0,107 & 0,001 & $-0,029$ & 0,113 & $\mathbf{0 , 2 7 5}$ & 0,070 & 0,121 \\
\hline & & $-0,045$ & 0,039 & $-0,032$ & 0,073 & $-0,132$ & 0,196 & \\
\hline & & & 0,085 & 0,053 & 0,071 & 0,010 & & \\
\hline
\end{tabular}

Grupo normal

\begin{tabular}{|l|l|l|l|l|l|l|l|l|}
\hline & & & 0,364 & 0,286 & 0,308 & 0,436 & & \\
\hline & 0,258 & 0,324 & 0,287 & 0,337 & 0,309 & 0,399 & \\
\hline & 0,228 & 0,440 & 0,477 & 0,322 & 0,440 & 0,316 & 0,000 & 0,461 \\
\hline 0,368 & 0,345 & 0,481 & 0,363 & 0,397 & 0,375 & 0,561 & & 0,390 \\
\hline 0,262 & 0,334 & 0,434 & 0,444 & 0,334 & 0,426 & 0,478 & & 0,363 \\
\hline & 0,482 & 0,395 & 0,364 & 0,460 & 0,483 & 0,507 & 0,520 & 0,313 \\
\hline & & 0,238 & 0,305 & 0,388 & 0,394 & 0,136 & 0,344 & \\
\hline & & & 0,236 & 0,388 & 0,278 & 0,416 & & \\
\hline
\end{tabular}

Grupo glaucoma pre-perimétrico

\begin{tabular}{|l|l|l|l|l|l|l|l|l|}
\hline & & & 0,095 & 0,094 & 0,055 & 0,000 & & \\
\hline & 0,007 & $-0,063$ & $-0,039$ & $-0,029$ & $-0,027$ & $-0,045$ & \\
\hline & 0,066 & $-0,029$ & $-0,043$ & $-0,075$ & $-0,056$ & 0,028 & 0,025 & $-0,025$ \\
\hline$-0,032$ & 0,096 & $-0,034$ & $-0,035$ & $-0,018$ & 0,149 & $-0,071$ & & $-0,078$ \\
\hline$-0,030$ & 0,058 & 0,022 & $-0,022$ & 0,092 & 0,057 & $-0,009$ & & $-0,137$ \\
\hline & $-0,016$ & $-0,083$ & 0,126 & 0,014 & 0,045 & $-0,040$ & $-0,095$ & $-0,043$ \\
\hline & & 0,042 & $-0,155$ & 0,038 & $-0,032$ & $-0,058$ & $-0,012$ & \\
\hline & & & 0,040 & 0,043 & $-0,128$ & $-0,029$ & & \\
\hline
\end{tabular}

\section{Grupo HTO}

\begin{tabular}{|l|l|l|l|l|l|l|l|l|}
\hline & & $\mathbf{0 , 3 3 4}$ & $\mathbf{0 , 2 8 8}$ & $\mathbf{0 , 2 4 8}$ & $\mathbf{0 , 1 9 6}$ & & \\
\hline & $\mathbf{0 , 2 7 3}$ & $\mathbf{0 , 2 2 2}$ & $\mathbf{0 , 2 8 7}$ & $\mathbf{0 , 3 0 1}$ & $\mathbf{0 , 2 7 0}$ & 0,242 & \\
\hline & 0,215 & $\mathbf{0 , 2 7 0}$ & 0,215 & $\mathbf{0 , 3 4 5}$ & $\mathbf{0 , 2 6 0}$ & $\mathbf{0 , 2 3 7}$ & 0,186 & 0,181 \\
\hline 0,276 & $\mathbf{0 , 2 9 9}$ & $\mathbf{0 , 3 2 7}$ & $\mathbf{0 , 2 5 6}$ & $\mathbf{0 , 5 2 8}$ & $\mathbf{0 , 3 4 0}$ & $\mathbf{0 , 3 0 8}$ & & $\mathbf{0 , 3 1 3}$ \\
\hline 0,065 & 0,013 & 0,036 & 0,022 & 0,089 & 0,080 & $\mathbf{0 , 1 6 4}$ & & 0,151 \\
\hline & $-0,002$ & 0,119 & 0,024 & 0,025 & 0,142 & 0,077 & $-0,023$ & $-0,002$ \\
\hline & & 0,061 & 0,015 & 0,027 & $-0,022$ & 0,052 & 0,078 & \\
\hline & & & 0,066 & 0,072 & $-0,038$ & 0,085 & & \\
\hline
\end{tabular}

\section{Grupo glaucomas}

Fig. 5: Representación gráfica de los coeficientes de correlación de Pearson obtenidos entre la línea de modulación de contorno temporal-inferior y los 52 puntos del campo visual en los 4 grupos de estudio. Las correlaciones significativas están resaltadas en negrita.

que el área del anillo y el área del disco fueron los únicos parámetros que mostraron una asociación estadísticamente significativa con los índices globales de la perimetría automatizada, el primero de ellos se relaciona tanto con la DM como con la DSM, mientras que el segundo lo hace únicamente con la DM. Shuman y colaboradores (18), evaluaron la correlación estructura-función con el HRT y OCT, y encontraron que en conjunto la asociación era pobre pero el parámetro que mostró una relación mejor fue el volumen del anillo neurorretiniano, coincidiendo con los resultados de Tsai (19).

En nuestro estudio, los mejores indicadores estructurales de desarrollo de enfermedad en rela- ción con la DM, fueron los cocientes excavación/disco y anillo/disco. Para la DSM fue el área del anillo. En cuanto al número de puntos alterados en los sectores del campo visual, el nasalsuperior resultó ser el más relacionado con los parámetros estructurales del HRT, coincidiendo con otros autores como Kanamori (20), Nouri-Mahdavi (21), Ferreras (22), que han señalado que la lesión glaucomatosa suele comenzar con cambios en el polo inferior de la cabeza del nervio óptico que se traducen en defectos superiores en el campo visual. Otros parámetros que mostraron correlaciones moderadas fueron el espesor medio de la CFNR, la sección transversal de la CFNR (fundamentalmente 
con el número de puntos alterados con una probabilidad menor del $2 \%$ y $1 \%$ ) y las funciones discriminantes FSM y RB, especialmente esta última. No es de extrañar que las funciones discriminantes se encuentren entre los parámetros con mayor número y fuerza de asociaciones ya que incrementan la capacidad diagnóstica del aparato mediante la combinación de parámetros (23). Otros autores han encontrado que el área del anillo y la sección transversal de la CFNR son los mejores indicadores de cambios en la DM para el glaucoma (24).

Con respecto a las correlaciones con los valores umbral de los 52 puntos del campo visual, de nuevo fueron mayores en sujetos con glaucoma. En este grupo, los parámetros del HRT II que mejores correlaciones presentaron fueron con los valores umbral de los puntos del campo visual fueron: el área de excavación, el área de anillo, en cociente excavación/disco, el cociente anillo/disco, el volumen del anillo, el espesor medio de la CFNR, la sección transversal de la CFNR, los cocientes excavación/disco horizontal y vertical, las líneas de modulación del contorno temporal-superior y temporal-inferior, y las funciones discriminantes FSM y RB.

El grado de asociación obtenido no es suficiente como para permitir el diagnóstico precoz usando los parámetros de evaluación estructural de forma aislada, por tanto, combinando la información que ambas pruebas ofrecen, se puede mejorar la capacidad diagnóstica y de seguimiento de la enfermedad. No obstante, es importante conocer qué parámetros estructurales y funcionales tienen más peso y cómo se relacionan entre sí.

\section{BIBLIOGRAFÍA}

1. Kroese M, Burton H, Vardy S, Rimmer T, McCarter D. Prevalence of primary open angle glaucoma in general ophthalmic practice in the United Kingdom. Br J Ophthalmol 2002; 86: 978-980.

2. Foster P, Buhrmann R, Quigley H, Jonhson G. The definition and classification of glaucoma in prevalence surveys. Br J Ophthalmol 2002; 86: 238-242.

3. Kroese M, Burton H. Primary open glaucoma. The need for a consensus case definition. J Epidemiol Community Health 2003; 57: 752-754.

4. Gupta N, Weinreb RN. New definitions of glaucoma. Curr Oppin Ophthalmol 1997; 8: 38-41.

5. Coleman AL, Brigatti L. The glaucomas. Minerva Med 2001; 92: 365-379.

6. Garway-Heath DF, Poinoosawmy D, Wollstein G, Viswanathan A, Kamal D, Fontana L, et al. Inter-and intraob- server variation in the analysis of optic disc images: comparison of the Heidelberg retina tomograph and computer assisted planimetry. Br J Ophthalmol 1999; 83: 664-669.

7. Schunmugan M, Azuara-Blanco A. The quality of reporting of diagnostic accuracy studies in glaucoma using the Heidelberg retina tomography. Invest Ophthalmol Vis Sci 2006; 47: 2317-2323.

8. Bossuyt PM, Reitsma JB, Bruns DE, Gatsonis CA, Glasziou PP, Irwig LM, et al. The STARD statement for reporting studies of diagnostic accuracy: explanation and elaboration. Clin Chem 2003; 49: 7-18.

9. Chylack LT Jr, Wolfe JK, Singer DM, Leske MC, Bullimore MA, Bailey IL, et al. The Lens Opacities Classification System III. The Longitudinal Study of Cataract Study Group. Arch Ophthalmol 1993; 111: 831-836.

10. Hodapp E, Parrish RK, Anderson DR. Clinical Decisions in Glaucoma. St. Louis, MO: Mosby; 1993; 52-61.

11. Mikelberg FS, Parfitt CM, Swindale NV, Graham SL, Drance SM, Gosine R. Ability of the Heidelberg retina tomography to detect early glaucomatous visual field loss. J Glaucoma 1995; 4: 242-247.

12. Burk RO, Noack H, Rohrschneider K, Volcker HE. Prediction of glaucomatous visual fields defects by reference plane independent three-dimensional optic nerve head parameters, In: Wall M, Wild JM. Perimetry Update 1998/99: Proceedings of the XII International Perimetric Society Meeting, Gardone Riviera (BS), 1998. The Hague: Walter Kugler, 1999; 463-474.

13. Lopez-Peña MJ, Ferreras A, Polo V, Larrosa JM, Honrubia FM. Relación entre perimetría automatizada convencional y HRT, OCT y GDx en sujetos normales, hipertensos oculares y glaucomatosos. Arch Soc Esp Oftalmol 2007; 82: 197-208.

14. Iester M, Mikelberg FS, Courtright P, Drance SM. Correlation between the visual field indices and Heidelberg Retina Tomograph parameters. J Glaucoma 1997; 6: 78-82.

15. Brigatti L, Caprioli J. Correlation of visual field with scanning confocal laser optic disc measurements in glaucoma. Arch Ophthalmol 1995; 113: 1191-1194.

16. Uchida H, Brigatti L, Caprioli J. Detection of structural damage from glaucoma with confocal laser image analysis. Invest Ophthalmol Vis Sci 1996; 37: 2393-2401.

17. Lan YW, Henson DB, Kwartz AJ. The correlation between optic nerve head topographic measurements, peripapillary nerve fibre layer thickness, and visual field indices in glaucoma. Br J Ophthalmol. 2003; 87: 1135-1141.

18. Shuman JS, Wollstein G, Farra T, Hertzmark E, Aydin A, Fujimoto JG, et al. Comparison of optic nerve head measurements obtained by optical coherence tomography and confocal scanning laser ophthalmoscopy. Am J Ophthalmol 2003; 135: 504-512.

19. Tsai CS, Zangwill L, Sample PA, Garden V, Bartsch DU, Weinreb RN. Correlation of peripapillary retinal height and visual field in glaucoma and normal subjects. J Glaucoma 1995; 4: 110-116.

20. Kanamori A, Nakamura M, Escano MF, Seya R, Maeda $H$, Negi A. Evaluation of lagucomatous damage on retinal nerve fiber layer thickness measured by optical coherence tomography. Am J Ophthalmol 2003; 135: 513-520.

21. Nouri-Mahdavi K, Hoffman D, Tannenbaum DP, Law SK, Caprioli J. Identifying early glaucoma with optical coherence tomography. Am J Ophthalmol 2004; 137: 228-235. 
22. Ferreras A, Pablo LE, Pajarín AB, Larrosa JM, Polo V, Honrubia FM. Logistic regresión análisis for early glaucoma diagnosis using optical coherence tomography. Arch Ophthalmol 2008; 126: 465-470.

23. Ferreras A, Pablo LE, Larrosa JM, Polo V, Pajarín AB, Honrubia FM. Discriminating between normal and glau- coma-damaged eyes with the Heidelberg Retina Tomograph 3. Ophthalmology 2008; 115: 775-781.

24. Mistlberger A, Liebman JM, Greenfield DS, Pons ME, Hoh $S T$, Ishikawa $H$, et al. Heidelberg retina tomography and optical coherence tomography in normal, ocular hypertensive and glaucomatous eyes. Ophthalmology 1999; 106: 2027-2032. 